\title{
DESCRIPCIÓN DE LOS ESTADIOS NINFALES DE FROESCHNERIA PILIGERA (STÅL) (HEMIPTERA: HETEROPTERA: LYGAEOIDEA: RHYPAROCHROMIDAE: MYODOCHINI), SU DISTRIBUCIÓN EN MÉXICO Y NOTAS SOBRE SU BIOLOGIA
}

\author{
Luis Cervantes Peredo y Marcela Briceño Báez \\ Red de Biodiversidad y Sistemática, Instituto de Ecología, A.C. Carretera Antigua a Coatepec, \\ No. 351, El Haya, Xalapa, C.P. 91070. Veracruz. MÉXICO. E-mail: luis.cervantes@inecol.edu.mx
}

Cervantes Peredo, L. \& M. Briceño. 2010. Descripción de los estadios ninfales de Froeschneria piligera (Stål) (Hemiptera: Heteroptera: Lygaeoidea: Rhyparochromidae: Myodochini), su distribución en México y notas sobre su biología. Acta Zool. Mex. (n. s.), 26(3): 685-703.

RESUMEN. Se describen e ilustran en detalle todos los estadios de Froeschneria piligera (Stål), incluyendo notas sobre su biología, se le ha encontrado alimentándose de las semillas de varias especies vegetales, principalmente de especies de Asteraceae, y es una especie terrestre facultativa, alimentándose de las semillas de algunas especies de Ficus. Se crió en laboratorio utilizando semillas de girasol.

Palabras Clave: Ficus, Helianthus, Estadios.

Cervantes Peredo, L. \& M. Briceño. 2010. Description of the immature stages of Froeschneria piligera (Stål) (Hemiptera: Heteroptera: Lygaeoidea: Rhyparochromidae: Myodochini), its distribution in Mexico and notes on its biology. Acta Zool. Mex. (n. s.), 26(3): 685-703.

ABSTRACT. Descriptions and illustrations of all instars about Froeschneria piligera (Stål), and notes on its biology, it has been found feeing on the seeds of several plants, mainly species of Asteraceae, it is also considered a facultative terrestrial species, because it feeds on some species of Ficus. It was reared in the laboratory using sunflower seeds.

Key Words: Ficus, Helianthus, Instars

\section{INTRODUCCIÓN}

El género Froescheneria fue descrito por Harrington (1980), transfiriendo a, Ligyrocoris infumatus Distant, Ligyrocoris multispinus Stål, Ligyrocoris oblitus Distant, Ligyrocoris piligerus (Stål) y Pachybrachius vicinalis (Distant). El género incluye estas cinco especies, de las cuales $F$. oblitus y $F$. infumatus, se encuentran reportadas solo para Guatemala (Slater 1964), y F. multispinus, F. piligera, F. vicinalis para México; F. multispinus se distribuye en los estados de: Campeche, 
Chiapas, Guerrero, Michoacán, Morelos, San Luis Potosí y Veracruz; F. piligera se distribuye en los estados de: Durango, Guerrero, México, Morelos, México, Oaxaca, Tabasco, Veracruz y Yucatán; $F$. vicinalis se encuentra distribuida en los estados de: Colima, Jalisco y Veracruz (Slater \& Brailovsky 2000). Tanto el género Froeschneria como Lygirocoris presentan un estridulitrum en forma de media luna en el vientre abdominal, pero se diferencian ya que Froeschneria presenta una profunda impresión transversal pronotal, un anillo amarillo pálido en la parte basal del artejo antenal IV, y una hilera doble de espinas en el fémur anterior, y aunque Lygirocoris presenta también una profunda impresión pronotal, tiene un artejo antenal IV unicolor y solo presenta una hilera de espinas en el fémur anterior. Son pocas las notas acerca de la biología de las especies de Froeschneria, salvo los reportes de Alayo (1973), quien colecto F. piligera en Helianthus debilis Nutt. y Helianthus annuus L. y Cervantes (2005), que menciono que ninfas y adultos de la misma especie se alimentan de semillas de Ficus cotinifolia H.B.K.

En el presente trabajo se describe e ilustra en detalle todos los estadios de Froeschneria piligera, incluyendo notas acerca de su biología, plantas hospederas y registros de su distribución en México.

\section{MATERIAL Y MÉTODOS}

Para el presente trabajo se utilizaron ejemplares depositados en la Colección Nacional de Insectos, Universidad Nacional Autónoma de México (CNIN); en la Colección Entomológica del Instituto de Ecología, A. C. Xalapa Veracruz (IEXA) y en la Entomological Collection of the University of California, Riverside (UCRC), solamente se especifica en el material revisado los ejemplares de estas dos últimas colecciones, el resto pertenecen a CNIN.

Los ejemplares utilizados para la cría se obtuvieron en la localidad de Chazumba, Km 16 al Suroeste de Chazumba, en el estado de Oaxaca. Los ejemplares se colectaron utilizando aspirador. Los adultos se depositaron en contenedores de plástico $(9 \times 8 \mathrm{~cm})$. Se coloco en cada contenedor dos o tres semillas de girasol y un algodón humedecido, cada tres días se revisaron los contenedores, se cambiaron las semillas y se registró la presencia de huevos, eclosión, muda o muerte de los ejemplares. Se dibujó cada estadio con ayuda de una cámara clara y en las descripciones las medidas están dadas en milímetros.

Los colectores se abreviaron de la siguiente manera: H. Almada (H. A.); E. Aranda (E. A.); F. Arias (F. A.); R. Barba (R. B.); E. Barrera (E. B.); C. Bolivar (C. B.); H. Brailovsky (H. B.); K. Brailovsky (K. B.); D. Brzoska (D. B.); J. Bueno S. (J. B. S.); J. Butze (J. B.); A. Cadena (A. C.); R. Carranza (R. C.); L. E. Carroll; A. Casasola (C. S.); L. Cervantes (L. C.); J. L. Colin (J. L. C.); A. M. Corona (A. M. C.); A. Delgado (A. D.); E. Elizalde (E. E.); C. Estrada; F. Figueroa (F. F.); A. García (A. G.); M. García (M. G.); S. Gámez (S. G.); T. Henry (T. H.); A. Ibarra (A. I.); L. 
Lozada (L. L.); E. Mariño (E. M.); G. B. Marshall (G. B. M.); J. Márquez (J. M.); E. Martín; C. Mayorga (C. M.); V. Meléndez (V. M.); R. Mendoza (R. M.); L. F. de la Mora (L. F. de la M.); M. A. Morón (M. A. M.); G. Noguera (G. N.); A. L. Norrbom; T. Novelo (T. N); L. O`Brien; C. W. O`Brien; G. Ortega (G. O); I. Pacheco (I. P.); D. Pallister; J. C. Pallister (J. C. P.); D. Peláez (D. P.); A. Pérez (A. P.); J. Peña (J. P.); N. Peñaloza; J. Pinto; E. Ramírez (E. R.); P. Rauch; L. Rivera (L. R.); A. Rodríguez (A. R.); Rojas (R.); R. Schuh (R. S.); W. Shon (W. S.); V. H. Toledo (V. H. T); L. Torres (L. T.); H. Velasco (H. V.); M. Vertiz (M. V.); G. Wibner (G. W.); S. Zaragoza (S. Z.); R. Zurbia (R. Z.). Sin embargo, algunos colectores se mencionan completos porque se citan en pocas ocasiones.

\section{Froeschneria piligera (Stål)}

(Figs.1-8)

1857 Lygaeus (Beosus) abdominalis Guérin-Méneville, Hist. Phys., Polit., Nat. Cuba, 7: 397-398

1862 Plociomera piligera Stål, Stett. Ent. Zeit., 23: 312

1906 Ligyrocoris constrictus: Barber, Mus. Brook. Inst. Arts, Sci., Sci. Bull., 1: 275

1914 Ligyrocoris abdominalis: Barber, Bull. Am. Mus. Nat. Hist., 33: 512

1939 Perigenes constrictus: Glick, U.S. Dept. Agr. Tech. Bull., 673: 23

1964 Ligyrocoris piligerus: Slater, Cat. Lygaeidae World, 2: 1092

1973 Ligyrocoris piliger: Steyskal, Proc. Ent. Soc. Wash., 75: 278

1980 Froescheneria piligera: Harrington, Bull. Am. Mus. Nat. Hist., 167: 101

Huevo: Forma cilíndrica con la parte superior plana y la inferior ligeramente puntiaguda. Mide $1.19 \pm 0.03 \mathrm{~mm}$ de largo por $0.5 \pm 0.02 \mathrm{~mm}$ de ancho $(\mathrm{n}=10)$. La parte superior del huevo con seis pequeños procesos micropilares arreglados de manera circular. De color blanquecino al ser depositados, tornándose rojizos posteriormente. Los ojos aparecen como manchas de color rojo.

Primer estadio: Alargado ligeramente. Cabeza, pro-, meso- y metanoto, pleuras y glándulas senescentes de color pardo claro. Ojos rojos, antenas, patas y rostro, amarillo claro. Abdomen dorsal y ventralmente amarillo pálido. Rostro sobrepasa las metacoxas.

Mediciones $(\mathrm{n}=10)$. Longitud total del cuerpo $1.61 \pm 0.24$; longitud de la cabeza $0.45 \pm 0.08$; anchura a través de los ojos $0.45 \pm 0.03$; distancia interocular $0.31 \pm 0.02$; distancia postocular $0.17 \pm 0.29$; artejos antenales: I $0.14 \pm 0.02$, II $0.25 \pm 0.05$, III $0.24 \pm 0.03$, IV $0.41 \pm 0.03$; artejos rostrales: I $0.42 \pm 0.49$, II $0.28 \pm 0.05$, III $0.25 \pm 0.02$, IV $0.28 \pm 0.03$; longitud del pronoto $0.18 \pm 0.02$; anchura ángulos humerales $0.44 \pm 0.04$; anchura a través del margen anterior del pronoto $0.50 \pm 0.03$; pata posterior: longitud fémur $0.35 \pm 0.02$; longitud tibia $0.37 \pm 0.04$; longitud tarsos: I $0.09 \pm 0.04$, II $0.16 \pm 0.02$. 

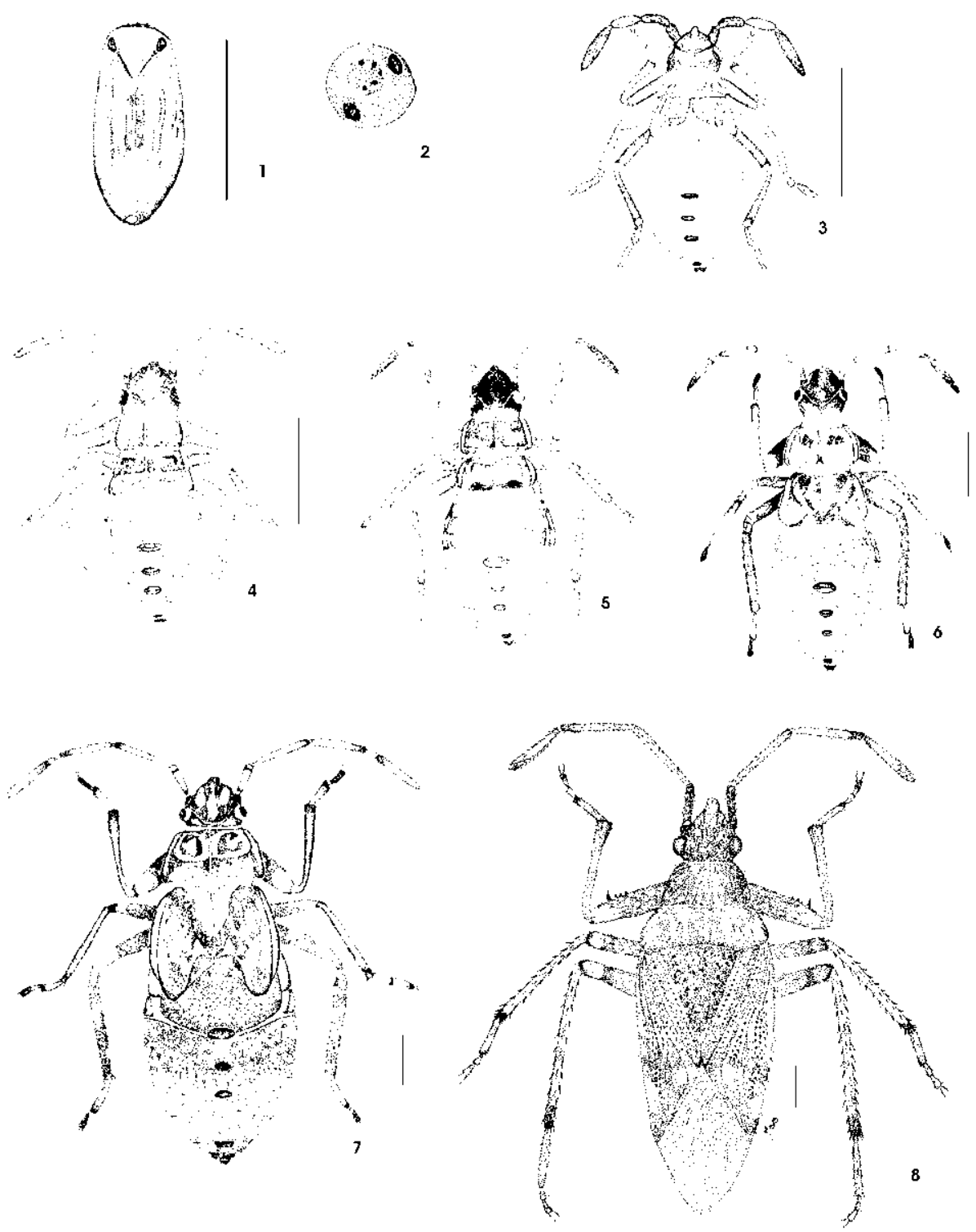

Figuras 1-8. Estadios de desarrollo de Froeschneria piligera (Stål). 1. Huevo vista lateral.

2. Huevo vista superior. 3. Primer estadio. 4. Segundo estadio. 5. Tercer Estadio. 6. Cuarto Estadio. 7. Quinto Estadio. 8. Adulto. 
Segundo estadio: Cabeza, pardo oscuro, ojos rojos. I, II y III artejos antenales amarillo pálido, IV artejo pardo oscuro; rostro, pro-, meso-, y metapleuras pardo claro. Pro-, meso-, y metanoto, pardo claro, este último con dos franjas pardo obscuro a cada lado de la línea media. Fémures pardo claro y tibias hasta el tercio anterior, amarillo pálido el resto de las tibias y tarsos del mismo color. Glándulas senescentes pardo oscuro. Abdomen pardo claro, presentando numerosos puntos de color blanco, en cada uno de los segmentos. Sutura en "Y" bien delimitada por una línea pardo claro. Glándulas senescentes entre los artejos III-IV, V-VI. Rostro sobrepasando las metacoxas.

Mediciones $(\mathrm{n}=10)$. Longitud total del cuerpo 2.64 \pm 0.14 ; longitud de la cabeza $0.55 \pm 0.45$; anchura a través de los ojos $0.61 \pm 0.28$; distancia interocular $0.40 \pm 0.17$; distancia postocular $0.14 \pm 0.02$; artejos antenales: I $0.20 \pm 0.02$, II $0.4 \pm 0.04$, III $0.35 \pm 0.03$, IV $0.52 \pm 0.02$; artejos rostrales: I $0.32 \pm 0.03$, II $0.38 \pm 0.03$, III $0.34 \pm 0.03$, IV $0.31 \pm 0.02$; longitud del pronoto $0.28 \pm 0.02$; anchura ángulos humerales $0.63 \pm 0.04$; anchura a través del margen anterior del pronoto $0.69 \pm 0.03$; pata posterior: longitud fémur $0.44 \pm 0.04$; longitud tibia $0.48 \pm 0.04$; longitud tarsos: I $0.14 \pm 0.02$, II $0.22 \pm 0.03$.

Tercer estadio: Cuerpo ligeramente alargado, alcanzando la anchura máxima en el IV segmento abdominal. Cabeza pardo oscuro, ojos rojos, artejos antenales I, II y III amarillo ocre, base del artejo I y artejo IV pardo claro. Pro- y mesonoto pardo claro, metanoto amarillo ocre, con dos bandas en cada lado de la línea media, pro-, meso- y metapleura, tibia posterior y espinas pardo oscuro; fémures pardo claro, tibia anterior y media amarillo ocre, coxas y abdomen alternando entre amarillo ocre y pardo claro, con puntuaciones blancas en todos los segmentos abdominales. Vientre abdominal amarillo ocre y pardo claro en los márgenes laterales. Glándulas senescentes pardo obscuro. Sutura en "Y" bien definida por una línea pardo claro. . Cabeza, tórax y abdomen presenta sedas opacas transparentes, el margen abdominal con sedas. Rostro alcanza metacoxas. Fémur anterior ligeramente ensanchado. Tibias anterior, media y posterior presentan una hilera de espinas recorriendo el margen interno y los márgenes laterales. Glándulas senescentes entre los segmentos III-IV, V-VI. Tricobotrios visibles, se observan en el cuarto segmento abdominal uno, en el quinto y sexto tres y en el séptimo dos. Espiráculos dorsales del segundo al cuarto segmento abdominal.

Mediciones $(\mathrm{n}=10)$. Longitud total del cuerpo 3.28 \pm 1.18 ; longitud de la cabeza $0.72 \pm 0.75$; anchura a través de los ojos $0.8 \pm 0.04$; distancia interocular $0.54 \pm 0.06$; distancia postocular $0.14 \pm 0.06$; artejos antenales: I $0.25 \pm 0.33$, II $0.57 \pm 0.03$, III $0.51 \pm 0.07$, IV $0.67 \pm 0.05$; artejos rostrales: I $0.44 \pm 0.04$, II $0.52 \pm 0.03$, III $0.44 \pm 0.4$, IV $0.40 \pm 0.06$; longitud del pronoto $0.48 \pm 0.08$; anchura ángulos humerales $0.84 \pm 0.04$; anchura a través del margen anterior del pronoto 1.02 \pm 0.02 ; pata posterior: longitud fémur $0.81 \pm 0.09$; longitud tibia $0.95 \pm 0.07$; longitud tarsos: I $0.15 \pm 0.15$, II $0.25 \pm 0.05$. 
Cuarto estadio: Alargado ligeramente, anchura máxima del cuerpo a la altura del IV segmento abdominal. Cabeza pardo oscuro, con la base amarillo ocre, ojos pardo oscuro y rojizo. Artejos antenales I, II, III amarillo ocre, base del artejo I pardo oscuro y margen distal del III y IV rojizo. Pronoto pardo claro con dos manchas pardo oscuro a cada lado de la línea media, con márgenes blancos con sus extremos pardos oscuros. Mesonoto y escutelo pardo claro, resto del mesonoto amarillo ocre. Pro-, meso- y metapleuras, fémures, glándulas senescentes y parte proximal de la tibias media y posterior pardo oscuro, coxas intercalando entre amarillo ocre y pardo oscuro, tibias anteriores amarillo ocre. Tarsos I amarillo ocre, tarsos II pardo claro. Vientre abdominal combinando pardo claro y amarillo ocre, los tres últimos segmentos presentan una mancha parda oscura en la línea media. Abdomen pardo claro con manchas blancas, más abundantes en los tres primeros segmentos. Sutura en "Y" bien diferenciada por una línea pardo claro. Presencia de sedas dirigidas hacia arriba, en cabeza y tórax, en abdomen hacia delante, el margen abdominal con sedas. Rostro alcanza las metacoxas. Glándulas senescentes entre los segmentos III-IV, VVI. Fémur anterior engrosado, presentando dos espinas. Tibias anterior, media y posterior presenta una hilera de espinas recorriendo el margen interno y los márgenes laterales. Tricobotrios visibles como en el estadio anterior.

Mediciones $(\mathrm{n}=1)$. Longitud total del cuerpo 5.65; longitud de la cabeza 1; anchura a través de los ojos 1.1; distancia interocular 0.7 ; distancia postocular 0.3 ; artejos antenales: I 0.4, II 0.85, III 0.75, IV 0.8; artejos rostrales: I 0.6, II 0.85, III 0.65 , IV 0.8 ; longitud del pronoto 0.8 ; anchura ángulos humerales 1.15 ; anchura a través del margen anterior del pronoto 1.45 ; longitud total del escutelo 0.76 ; anchura máxima de escutelo 0.84; pata posterior: longitud fémur 1.2; longitud tibia 1.15; longitud tarsos: I 0.3 , II 0.3 .

Quinto estadio: Cuerpo alargado, con la máxima anchura a través del III segmento abdominal. Cabeza pardo oscuro en los márgenes laterales, y pardo claro en los jugum y base del tylus. Base y ápice de los artejos antenales I, ápice del artejos antenales II y III, base el artejo antenal IV pardo oscuro y el resto pardo claro; ojos, glándulas senescentes abdominales, tibia posterior, vientre abdominal, pardo oscuro. Lóbulo anterior del pronoto presenta dos manchas, una a cada lado de la línea media, de color pardo oscuro, lóbulo posterior pardo claro, pardo oscuro y almohadillas alares presentan una combinación entre pardo oscuro y pardo claro, escutelo de color pardo oscuro en la parte lateral y pardo claro en el centro. Fémures pardo oscuro, y en la parte distal pardo claro. Tibias anterior y media, pardo claro, tarsos amarillo ocre; coxas intercalando colores entre el pardo oscuro y amarillo ocre; la parte más visible de los primeros segmentos abdominales, presentan puntos sobre un fondo pardo oscuro de las cuales sale un pelo. Resto de los segmentos abdominales con presencia de estos puntos pero en menor proporción. Márgenes laterales del abdomen con pelos oscuros. Sutura en "Y" bien delimitada por una banda pardo obscuro. 
Cabeza, tórax y abdomen, con pelos pardo oscuro opacos. Presencia de sedas dirigidas hacia arriba, en cabeza y tórax, en abdomen hacia delante, el margen abdominal con sedas. Ojos sobresalientes, I artejo antenal mas corto, II artejo más largo, artejos III y IV casi de la misma longitud. Rostro alcanza metacoxas. Márgenes laterales del lóbulo anterior y posterior del pronoto rectos. Fémur anterior engrosado, presentando dos espinas. Tibias anterior, media y posterior con una hilera de espinas recorriendo el margen interno y los márgenes laterales. Tricobotrios visibles como en los estadios anteriores.

Mediciones $(\mathrm{n}=5)$. Longitud total del cuerpo 7.26 \pm 0.70 ; longitud de la cabeza $0.96 \pm 0.15$; anchura a través de los ojos $1.26 \pm 0.09$; distancia interocular $0.80 \pm 0.06$; distancia postocular $0.28 \pm 0.13$; artejos antenales: I $0.56 \pm 0.05$, II $1.26 \pm 0.11$, III $1.06 \pm 0.09$, IV 1.2 \pm 0.07 ; artejos rostrales: I $0.8 \pm 0.07$, II $0.96 \pm 0.05$, III $0.8 \pm 0$, IV $0.5 \pm 0$; longitud del pronoto $0.98 \pm 0.15$; anchura ángulos humerales $1.34 \pm 0.09$; anchura a través del margen anterior del pronoto $1.94 \pm 0.13$; longitud total del escutelo 1.3 \pm 0.13 ; anchura máxima de escutelo $1.32 \pm 0.18$; pata posterior: longitud fémur 1.69 \pm 0.14 ; longitud tibia 1.68 \pm 0.14 ; longitud tarsos: I $0.55 \pm 0.08$, II $0.43 \pm 0.05$.

Adulto: Cuerpo alargado, cubierto de numerosas sedas erectas, con los márgenes laterales del pronoto redondeados. Pronoto marcadamente bilobulado, con una incisión transversa muy bien definida, collar pronotal presente, definido por una línea similar a un surco. Fémures anteriores pardo-oscuros, con la base y ápice amarillopálido, con dos hileras de espinas, la del margen externo con solo algunas pequeñas espinas visibles y la del margen interno con varias espinas grandes; fémures medios y posteriores amarillo-pálido con un anillo subapical pardo-oscuro. Tibias anterior, media y posterior como en los estadios anteriores.

Macho: Mediciones $(\mathrm{n}=10)$. Longitud total del cuerpo 7.88 \pm 0.29 ; longitud de la cabeza 1.16 \pm 0.14 ; anchura a través de los ojos 1.31 \pm 0.04 ; distancia interocular $0.80 \pm 0.06$; distancia entre ocelos $0.51 \pm 0.03$; distancia postocular $0.19 \pm 0.06$; artejos antenales: I $0.14 \pm 0.08$, II $1.63 \pm 0.16$, III $1.28 \pm 0.12$, IV $1.40 \pm 0.13$; artejos rostrales: I $0.97 \pm 0.08$, II $1.05 \pm 0.09$, III $0.85 \pm 0.07$, IV $0.6 \pm 0.04$; longitud del pronoto $1.53 \pm 0.11$; anchura ángulos humerales $1.33 \pm 0.07$; anchura a través del margen anterior del pronoto 2.26 \pm 0.21 ; longitud total del escutelo $1.82 \pm 0.24$; anchura máxima de escutelo 1.39 \pm 0.34 ; pata posterior: longitud fémur $2 \pm 0.15$; longitud tibia $1.99 \pm 0.13$; longitud tarsos: I $0.64 \pm 0.1$, II $0.22 \pm 0.05$; III $0.3 \pm 0.02$.

Hembra: Mediciones $(\mathrm{n}=10)$. Longitud total del cuerpo $7.80 \pm 0.70$; longitud de la cabeza 1.18 \pm 0.16 ; anchura a través de los ojos $1.32 \pm 0.16$; distancia interocular $0.8 \pm 0.06$; distancia entre ocelos $1.06 \pm 1.74$; distancia postocular $0.15 \pm 0.04$; artejos antenales: I $0.70 \pm 0.09$, II $1.44 \pm 0.19$, III $1.28 \pm 0.16$, IV $1.42 \pm 0.18$; artejos rostrales: I $0.86 \pm 0.07$, II $1.05 \pm 0.13$, III $0.80 \pm 0.11$, IV $0.6 \pm 0.70$; longitud del pronoto $1.56 \pm 0.19$; anchura ángulos humerales $1.31 \pm 0.13$; anchura a través del margen anterior del

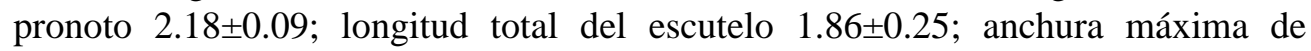


escutelo $1.31 \pm 0.16$; longitud fémur posterior $1.91 \pm 0.30$; longitud tibia posterior $1.98 \pm 0.18$; longitud tarsos posteriores: I $0.67 \pm 0.07$, II $0.02 \pm 0.04$; III $0.26 \pm 0.04$.

Notas biológicas: Ninfas y adultos fueron colectados alimentándose de semillas de Stuessya michoacana B. L. Turner \& F. G. Davis, en Melampodium sp. y Senecio prionopterus B. L. Rob \& Greenm (Asteraceae); también de semillas de Ficus cotinifolia, Ficus insipida Willd (Moraceae) y bajo las hojas de la palma, Dasylirum sp. (Liliaceae). Se encuentra asociada frecuentemente a Encinares, Bosques de Pino y Selva Baja. Algunos de los organismos son atraídos por la luz.

Como ya se mencionó anteriormente, ha sido reportada en Cuba asociada a Helianthus debilis, H. annuus (Alayo 1973). Cervantes (2005) mencionó que ninfas y adultos de la misma especie se alimentan de semillas de Ficus cotinifolia H.B.K.

Distribución: Estados Unidos (Henry \& Froeschner 1988; Slater \& Baranowski 1990), México (Slater \& Braiolvsky 2000), Islas del Caribe (Baranowski \& Slater 2005), Brasil, Guatemala, Panamá, Venezuela (Slater 1964), México: Campeche, Colima, Chiapas, Distrito Federal, Guerrero, Hidalgo, Estado de México, Michoacán, Morelos, Nayarit, Nuevo León, Oaxaca, Puebla, Querétaro, Quintana Roo, San Luis Potosí, Sinaloa, Sonora, Tabasco, Tamaulipas, Tlaxcala, Veracruz, Yucatán y Zacatecas.

Nuevos Registros: CAMPECHE: 62 ð, 28 우 Bolonchen de Rejón, 15-VI-1985, F. A.; 2 ㅇ, Calakmul, Ruinas Becan, 28-V-2008, L. C., C. M.; 1 ㅇ, Zona arqueológica

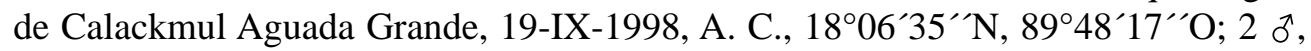
1 ㅇ, Km 178 Escárcega-Chetumal, 23-VI-1989, A. C., L. C.; 1 ㅇ, Escárcega, El Tormento, 18-VI-1989, A. C., L. C.; 2 ㅇ, Castamay, 15-VI-1985, M. V., H. V.; 2 ㅇ, Grutas Xtacumbilchunan, 20-VI-1989, A. C. L. C.; 1 ðત, 22 mi Campeche, 5-VIII1974, G. W. L. O’Brien. COLIMA: 1 q, La Salada, 20-X-1977, H. B.; 1 ㅇ, Km 17 Colima-Manzanillo, 19-X-1977, E. B.; 1 đ̋, 3 Km SE Ixtlahuacán, La Toma, 18-II2006, S. Z., $18^{\circ} 59^{\prime} 10.2^{\prime} \mathrm{N}, 103^{\circ} 42^{\prime} 13.5^{\prime} \mathrm{W}, 101 \mathrm{~m}$. CHIAPAS: $1{ }^{\jmath}$, Cintalapa de Figueroa, 13 mi NE Cintalapa, 01-VIII-1974, W. O’Brien, L. O’Brien, G. B. M., (IEXA); 1 ठ, Ocosingo, Chajul, Reserva Montes Azules, 10-16-VII-1987, F. A, R. B., L. C.; 2 ㅇ, 2 ð, Ocosingo, Montes Azules, 9-11-VII-1987, L. C.; 4 ð, 3 우, Ocosingo, Chajul, Reserva Montes Azules, 10-16-VII-1987, F. A., R. B.; 2 ð, 1 ㅇ, Ocosingo Km 7, Ocosingo-San Cristóbal, 27-VI-2007, C. M., G. O., L. C., $16^{\circ} 50^{\prime} 33^{\prime \prime} \mathrm{N}, 92^{\circ} 06^{\prime} 15^{\prime} \mathrm{W}, 1180 \mathrm{~m}$, Bosque Pino-Encino; $2{ }^{\star}$, Ocosingo Km 20 Ocosingo-Altamirano, C. M, G. O, L. C., $16^{\circ} 48^{\prime} 45^{\prime} \mathrm{N}, 9^{\circ} 02^{\prime} 38^{\prime} \mathrm{W}, 1077 \mathrm{~m}$, Bosque Pino-Encino; 1 ㅇ, Laguna de Montebello, VII-1978, E. B.; 1 우 Km 80 Palenque-Bonampak, 21-X-1980; 2 ठ, 1 ㅇ, Chorreadero, 5-IV-1979, H. B.; 3 ठ, 6 , Cañón del Sumidero (Mirador), 28-VIII-1978, G. O., E. B.; 1 ð 4 , + , Km 22 Cañón del Sumidero, 25-VII-1978, H. B.; 1 ð, Reserva el Ocote, 7-III-1993, G. O., E. J. G.; 4 ð, 2 ㅇ, Reserva el Ocote, 8-III-1993, G. O., E. B.; 2 ð, 1 ㅇ, Reserva el Ocote, 9III-1993, G. O., E. B.; 1 ðૈ, Reserva el Ocote, 2-10-XII-1993, G. O., E. B., A. C.; 1 
9 , Ocozocoautla, Reserva el Ocote-Nuevo, San Juan Chamula, 21-III-2007, C. M., G. O., L. C., 16 $52^{\prime} 54^{\prime \prime} \mathrm{N}, 93^{\circ} 28^{\prime} 32^{\prime \prime} \mathrm{W}, 836 \mathrm{~m}$; 1 o', $17 \mathrm{Km}$ E Ocozocoautla, 30-VI1981, J. Pinto, (UCRC); 1 오, Pueblo Soluchistan, Rincón Chamula, 26-VI-2007, C. M., G. O., L. C., $17^{\circ} 11^{\prime} 40^{\prime}{ }^{\prime} \mathrm{N}, 92^{\circ} 55^{\prime} 27^{\prime}$ W, 1769m; $1 \delta^{\nwarrow}$, Pueblo Nuevo Soluchistan

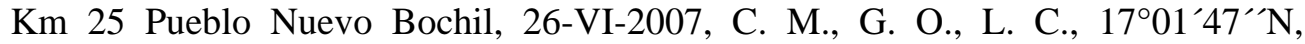
92 50`46”W, 1742m; 2 ð, 2 ㅇ, Pueblo Nuevo, Soluchistan Km 5 Pueblo NuevoJitotol, 26-VI-2007, L. C., G. O., C. M., $11^{\circ} 08^{\prime} 33^{\prime \prime} \mathrm{N}, 92^{\circ} 53^{\prime} 16^{\prime \prime} \mathrm{W}, 1624 \mathrm{~m}$, Bosque de Pino; 1 ð, Km 41 Motozintla-Comitán, 9-VII-1988, L. C.; 17 ð, 6 o , Frontera Comalapa Km 14 Frontera-Comitán, 19-III-2007, C. M., G. O., L. C., 1541’32”N, 92 ’03`43“'W, 799m; 1 ð’, Km 20 Motozintla-Comitán, 8-VIII-1988, A. C., L. C.; 1 ․, La Esperanza, 1500 mts, II-1927-IX-1939, D. P.; 1 ㅇ, Chinkultik, 2-VIII-1975, A. G. A; 1 q, San Cristóbal de las Casas, IX-1943, D. P.; 1 \&, El Salvador Urbina, Municipio Ángel A. Corzo, 7-VII-1993, S. Z., E. G., C. M., 850m; 2 ð, 2 ㅇ, Rosendo Salazar, 12-VII-2009, L. F. de la M.; 3 , 5 , $15 \mathrm{Km}$ del Aeropuerto de Tuxtla Gutiérrez camino a Villa Flores, 11-VII-2009, L. F. de la M., 16³0'518”N,

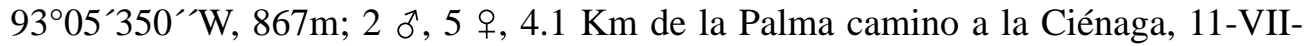

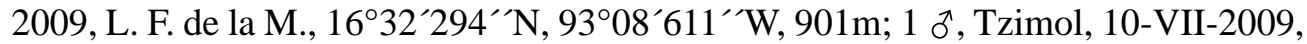
L. F. de la M., $16^{\circ} 11^{\prime} 870^{\prime \prime} \mathrm{N}, 92^{\circ} 11^{\prime} 582^{\prime \prime} \mathrm{W}, 1375 \mathrm{~m}$; 1 옹, Bonampak, 21-V-1980, H. B.; 2 ㅇ, Km 26 Revolución Mexicana-Cuxtepeques, 11-VII-1988, A. C., L. C., 600m; 2 ㅇ, $1 \mathrm{Km} \mathrm{S}$ de Ixtapa, carretera 195, 13-VIII-1993, C. M., 1000m; 1 우, Tapachula, Río Monte Perla, 19-III-1985, M. V.; 1 \&, Km 84 Tuxtla Gutiérrez, Villa Flores, 12-VII-1988, A. C., L. C., 530m; 13 ð, 18 오, Pichucalco, Km 5 a

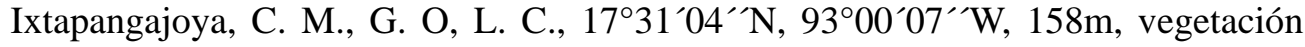
secundaria; 1 ㅇ, Reserva el Ocote, 3-V-1993, E. B.; 1 q, Ixhuatan, Km 3 carretera Pozo Rosario, 12-XII-1985, F. A., R. B., L. C.; 1 ㅇ, Unión Juárez, NW of Unión Juárez, S slope Volcán Tacana, Chiquihuites, 02-05-XI-1994, A. L. Norrbom, L. E. Carroll, C. Estrada, (IEXA); DISTRITO FEDERAL: 1 ð, Nepantla, 12-IV-1975, A. G. GUANAJUATO: 1 q, 5 Km Sur de Irapuato, 10-IV-2002, H. B., E. B.; 1 ㅇ, Marroquin Km 20 Querétaro-Celaya, 9-XI-2006, H. B., E. B.; 1 ð, Guanajuato, 10X-1981, H. B.; 1 ð, Jaral del Progreso, Cerro de Culiacán, 25-X-2007, H. B., L. C., $20^{\circ} 19^{\prime} 32^{\prime \prime} \mathrm{N}, 100^{\circ} 59^{\prime} 53^{\prime \prime} \mathrm{W}, 2095 \mathrm{~m}$, Matorral Espinoso; $1 \mathrm{o}^{\star}$, Jaral del Progreso,

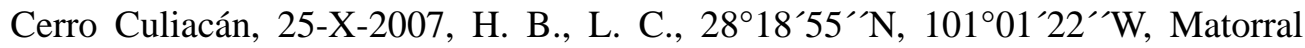

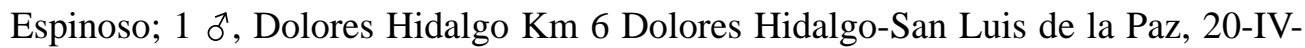

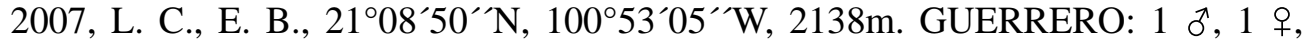
Coyuca de Catalán, Km 32 Coyuca-Zihuatanejo, 17-XI-2006, G. O., L. C., $18^{\circ} 70^{\prime} 34^{\prime \prime} \mathrm{N} 100^{\circ} 54^{\prime 2} 26^{\prime \prime} \mathrm{W}, 453 \mathrm{~m}$; 1 ㅇ, Km 100 Coyuca de Catalán-Zihuatanejo, 6VI-1984, E. B., H. B.; 1 ð , 1 ㅇ, Coyuca de Catalán Km 100 Coyuca-Zihuatanejo, 17-

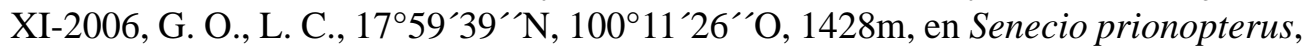
encinar; 6 o, 5 ㅇ, Zihuatanejo, Km 52 Zihuatanejo-Coyuca de Catalán, 20-XI-2006, $17^{\circ} 16^{\prime} 17^{\prime} \mathrm{N}, 99^{\circ} 32^{\prime} 58^{\prime} \mathrm{W}, 697 \mathrm{~m} ; 3$ o $^{`}, 9 \mathrm{Km}$ al S de Coyuca de Catalán, 17-X-2005, 
E. B., $18^{\circ} 16^{\prime} 50^{\prime} \mathrm{N}, 100^{\circ} 38^{\prime} 23^{\prime \prime} \mathrm{W}, 263 \mathrm{~m}$; 1 웅, Zihuatanejo, 2-VIII-1971, H. B.; 1 , Zihuatanejo Km 69 Zihuatanejo-Coyuca de Catalán, 18-XI-2006, G. O., L. C., $17^{\circ} 57^{\prime} 44^{\prime \prime} \mathrm{N}, 101^{\circ} 14^{\prime} 02^{\prime \prime} \mathrm{O}, 1662 \mathrm{~m}$; 1 우, Km 21 Coyuca de Catalán-Zirandaro, 14II-1984, E. B.; 1 o, Km 9 Chilpanzingo-Omilteme, 26-XI-1981, H. B., colecta nocturna; 3 ð, 2 ㅇ, Omilteme, 5-V-1982, H. B.; 1 , Omilteme, 16-V-1911, E. Martin, 1900m; 1 ð, 3 ㅇ, Km 16 Chilpancingo-Omilteme, 28-I-1982, A. I., M. G., H. B.; 1 ㅇ, Km 25 Chilpancingo-Omilteme, 26-V-1986, H. B.; 1 ð, $6 \mathrm{Km}$ Chilpancingo-Omilteme, 26-V-1986, H. B.; 1 ð, Omilteme, 9-XII-1981, H. B.; 1 ㅇ, Tecpan, 10-VIII-1971, H. B.; 1 ठ, Tecpan, 16-VIII-1971, H. B.; 1 우 Tecpan de Galeana, Piedra de Tlacoyunque Km 50 Acapulco-Zihuatanejo, 19-XI-2006, G. O., L. C., C. M., $17^{\circ} 15^{\prime} 34^{\prime \prime}$ N, 100 $59^{\circ} 55^{\prime \prime} \mathrm{W} ; 7$ ð`, 4 ㅇ, Tecpan de Galeana, Piedra de Tlacoyunque, Km 150 Acapulco-Zihuatanejo, 19-XI-2006, G. O., L. C., C. M., $17^{\circ} 15^{\prime} 34^{\prime \prime} \mathrm{N}, 100^{\circ} 59^{\prime} 55^{\prime} \mathrm{W}, 6 \mathrm{~m}$, en Stuessya michoacana; 1 ठ Tuxpan, 13-XII-1982, E. B., H. B.; 4 ㅇ, Iguala, Estación de Microondas, Tuxpan, 19-

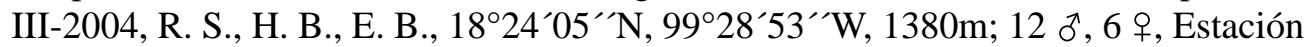
Microondas, Tuxpan, 4-II-1989, E. B., L. C., A. C., 1750m; 1 ð, Iguala, Estación Microondas, Tuxpan, 19-III-2004, R. S., H. B., L. C., 18 $29^{\prime} 47^{\prime \prime} \mathrm{N}, 99^{\circ} 21^{\prime} 01^{\prime \prime} \mathrm{W}$,

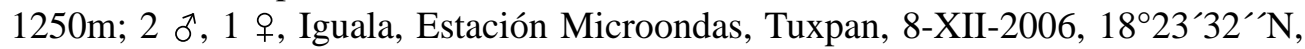

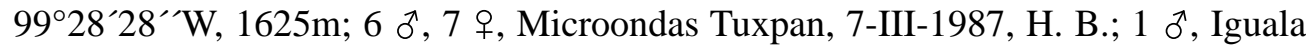

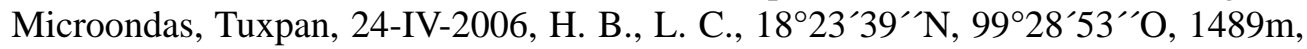
bajo Dasylirium sp; 1 ठ, Acuitlapa, V-1944, D. P.; 45 ðત, 34 ㅇ, Acatempan, 4-II-1989, E. B., A. C., L. C., 1675m; 1 ð, Tlapa Km 44 Tlapa-San Luis Acatlan, 14-VI-2006, H. B., L. C., $17^{\circ} 17^{\prime} 52^{\prime \prime} \mathrm{N}, 9^{\prime} 38^{\prime} 19^{\prime \prime} \mathrm{W}, 2162 \mathrm{~m}$, encinar; 1 ㅇ, $14 \mathrm{Km}$ al N de San Luis Acatlan, 13-VIII-2005, E. B., 16 $6^{\circ} 54^{\prime} 23^{\prime \prime} \mathrm{N}, 98^{\circ} 44^{\prime} 29^{\prime \prime} \mathrm{W}, 400 \mathrm{~m}$; 7 ð。, 6 ㅇ, San Luis Acatlan, 22-IX-2005, L. C., R. C., 16 $47^{\prime} 33^{\prime \prime} \mathrm{N}, 98^{\circ} 44^{\prime} 28^{\prime \prime} \mathrm{W}, 415 \mathrm{~m}$ en Melampodium sp; 7 ð, 8 ㅇ, Atlixtac Km 39 Chilapa-Tlapa, 11-XII-2006, L. C., C. M., $17^{\circ} 35^{\prime} 47^{\prime \prime} \mathrm{N}, 9^{\circ} 01^{\prime} 06^{\prime \prime} \mathrm{W}, 1425 \mathrm{~m}$, encinar; $1 \delta^{ð}$, Apaxtla Km 35 LiberaltepecSan Felipe del Ocote, 12-IX-2006, L. C., L. L., $18^{\circ} 11^{\prime} 31^{\prime \prime} \mathrm{N}, 100^{\circ} 02^{\prime} 45^{\prime \prime} \mathrm{W}, 1152 \mathrm{~m}$; 1 §, 1 ㅇ, Apaxtla, 5-III-1987, E. B., L. C.; 1 ð’, Tlalixtaquilla, Km 4, La Luz Juárez,

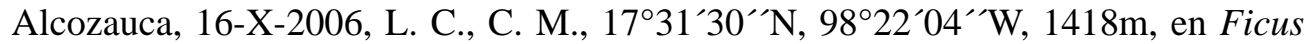
cotinifolia; 3 ठ, 2 ㅇ, Alcozauca, IX-1984, F. F.; 2 ð, 4Km N de Acuitlapan, H. B., E. B.; $18^{\circ} 36^{\prime} 18^{\prime \prime} \mathrm{N}, 99^{\circ} 32^{\prime} 02^{\prime \prime} \mathrm{W}, 1405 \mathrm{~m} ; 3{ }^{\jmath}, 12 \mathrm{Km}$ al NE de Alcozauca rumbo a Tlapa, 29-V-2009, $17^{\circ} 28^{\prime} 57^{\prime \prime} \mathrm{N}, 98^{\circ} 23^{\prime} 31^{\prime \prime} \mathrm{W}$, Pino-Encino; 1 ð’, 9 ㅇ, Km 20 carretera Arcelia-Teloloapan NE, E. B., 18 $22^{\prime} 06^{\prime \prime} \mathrm{N}, 100^{\circ} 09^{\prime} 51^{\prime \prime} \mathrm{W}, 696 \mathrm{~m} ; 1$ ○े, 2 , Km 10 Arcelia-Teloloapan, 28-X-2000, H. B., E. B., 850 m; 1 ㅇ, Acapulco, 10-IV1993, G. G.; 1 ㅇ, Acapulco, 21-XII-2003, H. B.; 1 ðð, Tlapa Km 5 Tlapa-Alpoyeca,

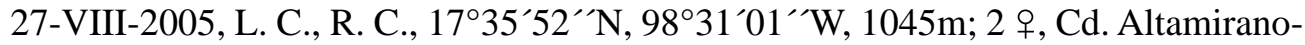
Arcelia Km 4 desviación a Nueva Filadelfia y el Tezontle, 19-X-2005, E. B., $18^{\circ} 17^{\prime} 07^{\prime \prime} \mathrm{N}, 100^{\circ} 29^{\prime} 50^{\prime \prime} \mathrm{W}, 453 \mathrm{~m}$; 1 o $^{\rtimes}, 2$ 우, Xalostoc, Iguala, 11-VII-1975, H. B.;

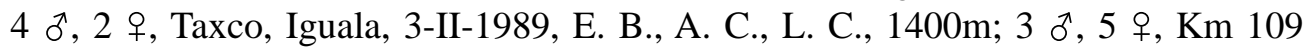


Taxco-Iguala, 3-II-1989, E. B., A. C., L. C.; 2 ð, 1 ㅇ, Km 10 Iguala-Taxco, 19-I1990, H. B., E. B.; 1 ㅇ, Km 145 Iguala-Altamirano, 23-XI-1984, F. A.; 1 ð, 1 ㅇ, Km 93 Iguala-Altamirano, 23-X-1984, F. A.; 2 ㅇ, Km 33 Iguala-Teloloapan, 28-XII1994, E. B., $18^{\circ} 24^{\prime} 88.8^{\prime}{ }^{\prime} \mathrm{N}, 42^{\circ} 52^{\prime} 52.7^{\prime \prime} \mathrm{W} ; 9$ ð`, 1 의, Iguala Km 10 Iguala-San José

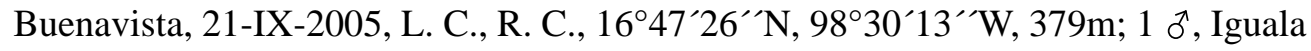
San Juan de la Unión, 12-XI-2005, L. C., $18^{\circ} 25^{\prime} 25^{\prime \prime} \mathrm{N}, 99^{\circ} 36^{\prime} 48^{\prime \prime} \mathrm{O}, 1115 \mathrm{~m} ; 3$ ð ${ }^{\rtimes}, 1$ + , Km 73 Cuernavaca-Taxco, 2-II-1989, L. C., E. B.; 1 q, El Huizteco, Taxco, III-

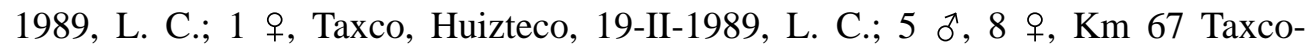
Alpuyeca, 20-XI-1984, M. V., H. V.; 2 , Km 67 Taxco-Alpoyeca, 24-XI-1984, M. V., F. A; 1 ð, 1 q, Km 8 Taxco-Acalpulco, 10-XII-1988, E. B., J. B. S., H. B.; 1 ㅇ, 4 Km Cacahuamilpa-Taxco, 21-XI-2003, $18^{\circ} 39.1^{\prime} \mathrm{N}, 99^{\circ} 30.2^{\prime} \mathrm{W}, 1200 \mathrm{~m} ; 1$ ð。, Km 7 Cacahuamilpa-Taxco, 12-06-2001, E. B., H. B.; 1 क , Teacalco, 2-II-1989, E. B., A. C., L. C., 1100m; 1 ð, Teucizapan, 21-X-1988, E. B, J. B. S., H. B.; 2 ð, D. Tetitlan, 22-X-1988, L. B., E. B., H. B.; 2 §, 1 ㅇ, Teucizapan, 3-II-1989, E. B., A. C., L. C.;

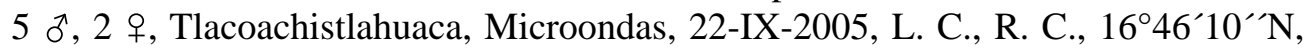
98 $18^{\prime} 06^{\prime \prime} \mathrm{W}, 418 \mathrm{~m}$ en Melampodium sp; 2 ð, Tlalpa Km 12 Tlalpa-Chilapa, 18-X-

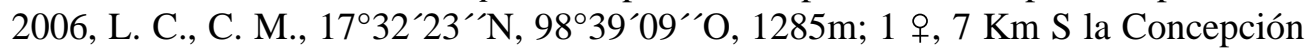

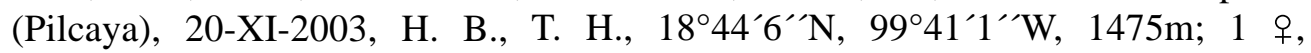
Chilpancingo, 28-VIII-1993, E. B., H. B.; 4 o, Cacahuamilpa, 7-X-2000, H. B., L. C.; 2 오, Chilpancingo, 20-I-1990, H. B.; 3 ๙, 2 ㅇ, Chilpancingo, Km 10

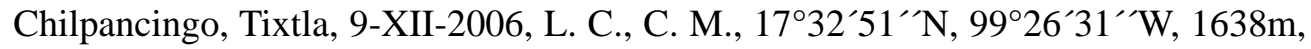
encinar; 1 $\sigma^{\star}$, Chilapa Km 8 Chilapa-Santa Cruz, 28-VIII-2005, L. C., R. C., $17^{\circ} 38^{\prime} 04^{\prime \prime} \mathrm{N}, 98^{\circ} 06^{\prime} 16^{\prime \prime} \mathrm{W}, 1619 \mathrm{~m}$ en Dasylirium sp; 1 ð, 4 ㅇ, Huizteco, 11-III1998, E. B., H. B.; 1 ð, 1 ㅇ, Playa Azul, 27-VIII-1985, R. B.; 2 ð, Rodecia, 11-VIII1971, H. B.; 9 ð, 1 ㅇ, Zihuaquio, 15-IV-1988, L. C., M. G., A. C., 1 ㅇ, Km 58 Milpalta-Oaxtepec, 27-I-1982, H. B.; 2 ठ, 1 ð, Km 5 Pilcaya-Despoblado, 18-III2004, R. S, H. B.; L. C.; 1 §, 1 ㅇ, San Miguel a 4 Km a E de Ajuchitlan del Progreso, 18 -X-2005, E. B., $18^{\circ} 08^{\prime} 51^{\prime \prime} \mathrm{N}, 100^{\circ} 25^{\prime} 50^{\prime \prime} \mathrm{W}, 310 \mathrm{~m}$; $1 \mathrm{o}^{\jmath}$, San Marcos Llano

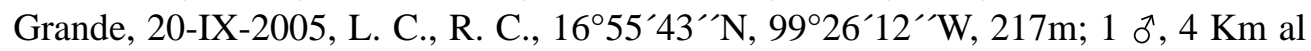
$\mathrm{W}$ de Malinaltepec (43 Km al $\mathrm{W}$ de Tlapa), 27-V-2006, E. B., $11^{\circ} 18^{\prime} 16^{\prime \prime} \mathrm{N}$, 98 $38^{\prime} 33^{\prime \prime} \mathrm{O}, 2260 \mathrm{~m}$; 1 ㅇ, Tierra Colorada camino a la Palma, L. C., R. C.,

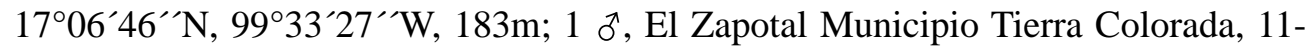
VIII-2005, E. B., $17^{\circ} 72^{\prime} 23^{\prime} \mathrm{N}, 9^{\circ} 32^{\prime} 19^{\prime} \mathrm{W}, 1518 \mathrm{~m}$, Selva Baja; 2 ơ $^{\star} 1$ 우 Zumpango del Río Km 6.5, desviación a Filo de Caballo, 25-VIII-2005, L. C., R. C., $17^{\circ} 47^{\prime} 38^{\prime \prime} \mathrm{N}, 99^{\circ} 34^{\prime} 47^{\prime \prime} \mathrm{W}, 906 \mathrm{~m}$; 1 ð`, Zumpango del Río Km 30 Chilpancingo-

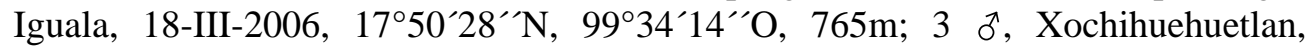

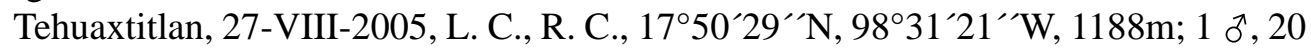
$\mathrm{Km}$ al $\mathrm{O}$ de Ometepec rumbo a Huixtepec, 12-VIII-2005, $16^{\circ} 39^{\prime} 29^{\prime \prime} \mathrm{N}$,

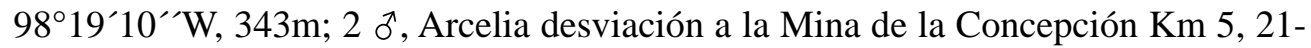
X-2005, E. B., $18^{\circ} 24^{\prime} 04^{\prime \prime} \mathrm{N}, 100^{\circ} 11^{\prime} 50^{\prime} \mathrm{W}, 660 \mathrm{~m}$; 1 으, Tlapa-Malinaltepec, $28 \mathrm{Km}$ 


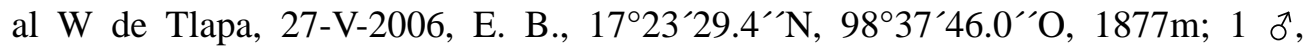
Chilpancingo, El Ocotito Km 5 desviación a Zoyatepec, 20-XI-2006, G. O., C. M., L. C., $17^{\circ} 16^{\prime} 17^{\prime \prime} \mathrm{N}, 99^{\circ} 34^{\prime} 58^{\prime \prime} \mathrm{W}, 723 \mathrm{~m}$, en Bosque de Pino; $1 \mathrm{o}^{\star}$, Marquelia Agua Zarca, 21-IX-2005, L.C., R. C., $16^{\circ} 38^{\prime} 03^{\prime}$ N $, 98^{\circ} 42^{\prime} 20^{\prime} \mathrm{W}, 272 \mathrm{~m}$; 1 ㅇ, $6 \mathrm{Km}$ al NE

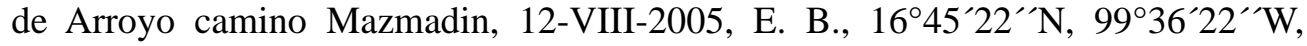
$323 \mathrm{~m}$.

HIDALGO: $1 \delta$, Otongo, 12-VII-1980, L. R., colecta nocturna; $2 \delta, 1$ ㅇ, Ixcuicuila, $13 \mathrm{Km}$ al SO de Huejutla, 11-V-1999, H. B., E. B.; 1 §, 2 ㅇ, Km 9

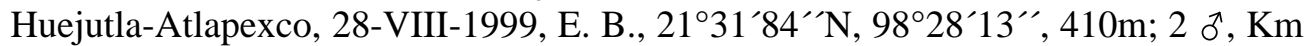
14 Huejutla-Atlapexco, 13-V-1999, H. B., H. B.; 3 , Km 8 desviación Huejutla de Reyes Atlapexco, 10-III-1999, H. B., E. B., $21^{\circ} 04^{\prime} 025^{\prime \prime} \mathrm{N}, 98^{\circ} 22^{\prime} 819^{\prime \prime} \mathrm{O}, 440 \mathrm{~m}$; 1 q Km 17 desviación Huejutla-Atencuapa, 11-III-1999, H. B., E. B., $21^{\circ} 02^{\prime} 087^{\prime}$ N , $98^{\circ} 22^{\prime} 547^{\prime \prime} \mathrm{O}$; 1 우, Huejutla, Xocotitla, 28-VIII-1999, E. B. $21^{\circ} 07^{\prime} 61^{\prime \prime} \mathrm{N}$,

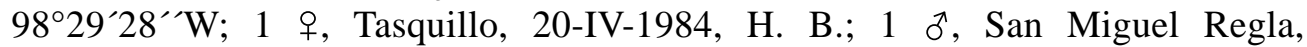
Tlaxocoyucan, 10-VI-1990, H. B., E. B.; 1 ð, Tecozautla, 29-IX-1999, H. B., E. B.; 1 ठ, Mecatlan, 26-XI-1998, E. B., H. B., 550 ft.; 1 ð, Huejutla, Atlapexco II, 6-VIII1999, E. B.; 1 , Zimapan, 19-IX-1999, H. B.; 1 ㅇ, Santiago de Anaya, 18-XII-1990, C. M., A. C., J. L. C. JALISCO: 1 ㅇ, Chamela, Estación Biológica, colecta nocturna, 15-XI-1978, H. B.; 1 ㅇ, Chamela, Estación Biológica, colecta nocturna, 17-III-1975, H. B.; 1 ð, Chamela, Estación Biológica, cuenca 1 TM 9149, 15-20-III-1992, A. R.; 2 đ, Chamela, Estación Biológica, 5-XI-1989, G. O., A. C.; 1 ㅇ, Chamela, Estación Biológica, El Tuito, 23-VII-1976, H, B.; 1 ㅇ, Chamela, 3-II-1993, H. B.; 1 ð, Chamela, 18-22-XII-1987, H. B.; 1 ㅇ, Chamela, 12-I-1975, H. B.; 1 đ, San BuenaVentura-Pueblo, 3-IV-1997, S. Z., 720m; 1 ð, San BuenaVentura-Pueblo, 6-II1997, S. Z., 720m; 1 오, San BuenaVentura-Pueblo, 8-I-1997, S. Z., 720m; 1 , San BuenaVentura-Pueblo, 9-II-1997, S. Z., 720m; 1 \&, Mismaloya, 2-VIII-1984, E. B.; 1 ㅇ, 1 ㅇ, Km 191 Ocotlán, Guadalajara, 26-XI-1988, A. C.; 1 ㅇ, Loc. 4 Amacahuatitlan, 6-VI-1997, S. Z.; 1 +, Hwy 54, 4 mi S Atenquique, 4500ft, 9-VIII1982, C. W. O’Brien, L. O’Brien, G. W.; 1 ․ Hwy 200, 4 mi S El Tuito, 1200ft, 10VIII-1982, C. W. O`Brien, L. O’Brien, G. W.; 1 ㅇ, Puerto Vallarta, 20-VII-1982, H. B.; 1 ð, Km 103 La Piedad-Guadalajara, 9-III-1988, H. B., L. C.; 1 क, Km 27 Tomatlán-La Cumbre, 18-IX-1988, E. R.; 1 오 Río San Nicolás, 3-III-1989, E. R. ESTADO DE MEXICO: 1 ㅇ, Malinalco, 9-XII-1978, E. B.; 12 ð。, 1 우, Malinalco, 12-I-1980, H. B.; 1 ㅇ, Malinalco, 8-VIII-1943, C. B.; 2 ð, 3 ㅇ, Malinalco, 19-XI1980, H. B., E. B.; 1 đ, Malinalco, 28-IV-1984, H. B.; 1 क, Malinalco, 26-VI-1980, H. B.; 1 q, 1 ð, Km 5 Malinalco-Tenancingo, 11-III-2003, H. B., E. B.; 1 ð, 1 ㅇ, Temascaltepec, Real de Arriba, 4-VI-1988, L. C.; 1 ð, Temascaltepec, 8-II-1979, E. B.; 3 ơ, 4 ㅇ, Km 10 Tejupilco-Temascaltepec, 20-X-1983, E. B.; 1 ㅇ, Km 87 TolucaTejupilco, 4-VI-1988, A. C.; 1 +, Zempoala, 6-I-1979, 2800 m, J. B. S.; 1 q, Milpa Alta, 9-IX-2009, H. B., E. B.; 1 ㅇ, Milpa Alta, 29-VII-1980, H. B.; đ,+ 1 ㅇ, Bejucos, 
desviación a Acamuchitlán, 16-XI-2006, G. O, C. M., L. C., 1851'31'”N, $100^{\circ} 16^{\prime} 42^{\prime \prime} \mathrm{W}, 928 \mathrm{~m} ; 13$ ðð, 8 옹, El Zapote, Acamuchitlan, 24-IX-1984, M. V., E. B., H. B., F. A.; 2 ð, 1 q, Sta. Bárbara 5 Km Poniente de Sto. Tomas de los Plátanos, 26II-1995, H. B, .E. B.; 1 ð, 1 ㅇ, Ameyuca, 4-X-2000, H. B., E. B.; 2 ㅇ, San Miguel, Ixtapa, 27-IX-1995, H. B., E. B.; 1 ð, Valle de Bravo, 3-4-VII-2004, W. S., H. B.; 1 ð, San Martín Otzoloapan, 27-II-1993, H. B., E. B.; 1 옹, Tonatico, 7-X-2000, H. B., E. B. MICHOACÁN: 2 ơ, Aguililla, 1-VIII-1985, F. A, R. B.; 1 đ, Tingambato, 20XI-1980, H. B.; 2 ठ, 4 ㅇ, La Huacana, 21-IX-1987, E. B., R. B., E. B.; 1 ઠ, El Limón, 21-IX-1987, E. M.; 1 ㅇ, Nueva Italia, 22-IX-1987. E. B.; 1 ð, Computo, 17IX-1987, E. B., R. B.; 3 ð, 1 q, Aputzio, 12-13-II-1984, E. B., H. B.; 2 ㅇ, 1 , Km 135 carretera Caleta de Campo-Manzanillo, 28-VIII-1985, F. A.; 1 q, Hwy 15, 19 mi. W. Jacona, 5400’' 13-VIII-1982, L. O’Brien, G. W; 1 ð, Turundero, 24-X-2001, H. B., E. B., 1840m; 1 ㅇ, San José, 9-11-1979, H. B.; 1 ð, 2 ㅇ, Km 98 Pátzcuaro-La Huacana, 1-VI-1988, A. C., L. C., 1200m; 1 ㅇ, Zitacuaro, Presa El Bosque, 23-VI1986, E. M.; 1 ð, El Laurel, 16-VII-1983, E. B.; 1 ð, Coahuayana, 11-II-1983, H. B.; 1 o, Pedernales, El Salitre, 19-IX-1987, E. B.; 1 ठ, Nueva Italia, 12-IX-1987, E. B.; 1 ð, Km 11 Las Cañas-Infiernillo, 19-IX-1989, R. B. MORELOS: 1 ㅇ, Yautepec, 20-X-1979, H. B.; 1 ð, Km 58 carretera México-Yautepec, 18-IX-1980, H. B.; 1 ð, Yautepec, 3-V-1991, H. B., E. B.; 1 ठ, Yautepec, 16-08-1997, H. B., E. B.; 7 ð, 3 , Tepoztlán, 20-II-1977, E. B.; 4 đ, 3 ㅇ, Tepoztlán, 25-II-1977, H. B.; 12 ð, 13 ㅇ, Tepoztlán, 3-IV-1981, H. B.; 1 đ̃, Tepoztlán, 28-IX-1945, C. B., H. B.; 1 ㅇ, Tepoztlán, 12-III-1974, H. B., 1900 m; 1 ㅇ, Tepoztlán, 14-III-1974, H. B.; 1 q, 1 ठ઼, Tepoztlán, 7-III-1974, H. B., 2105m; 1 ㅇ, Tepoztlán, 26-III-1974, H. B.; 1 o, Tlayacapan, San José de los Laureles, III-1996, J. M.; 18 ठ, 1 우 Tlayacapan, San José de los Laureles, I-1996, J. M.; 8 ठ઼, Tlayacapan, San José de los Laureles, XII1996, J. M.; 4 ð, Tlayacapan, San José de los Laureles, VII-1996, J. M.; 1 ðૈ, Tlayacapan, San José de los Laureles, XI-1996, J. M.; 1 ㅇ, Huautla 25 Km N 4 Km O estación CEAMISH, A. P., $16^{\circ} 27^{\prime} 67^{\prime \prime} \mathrm{N}, 9^{\circ} 02^{\prime} 475^{\prime \prime}$ O, 940m, T.L.3, Selva Baja Caducifolia; 1 ð, Tepalcingo CEAMISH subestación El Limón, 30-VIII-IX-2004, R.

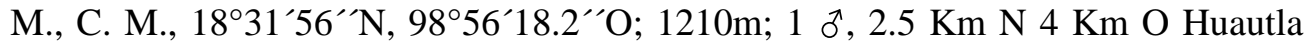
CEAMISH, 7-X-1996, S. Z., E. G., 940m; 2 ㅇ, Cuautla, 16-XII-1979, H. B.; 1 ㅇ, Cuautla, 23-XII-1981, H. B.; 1 +, Jonacatepec, 26-III-1981, H. B.; 1 , Jonacatepec, 26-III-1981, H. B.; 1 ㅇ, Jonacatepec de las Pilas, 5-II-2003, W. S.; 1 ㅇ, Jonacatepec, 19-IX-1984, H. B.; 2 ð, Amatlán, 20-III-2004, R. S, H. B., E. B., 1900`06”N,

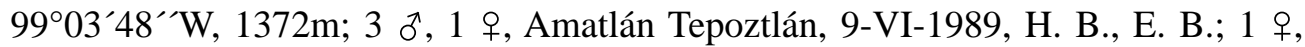
Cuernavaca, 11-III-1974, H. B., 2640m; 1 , Cuernavaca, 16-III-1974, H. B.; 1 o 2 , El Tepozteco, 13-II-1978, G. O.; 1 , Cañón de Lobos, 24-VIII-1976, E. B.; 2 đ, 3 Km S Acatipla, 7-I-1970, E. Marín; 4 ơ 2 q, Chacaltzingo, 11-XII-1986, E. B., H. B.; 1 ठ, Tepalcingo al $\mathrm{S}$ de el Limón, 23-25-VI-2006, V. H. T., A. M. C., $18^{\circ} 31^{\prime} 61.1^{\prime \prime} \mathrm{N}, 98^{\circ} 56^{\prime} 48.2^{\prime \prime} \mathrm{W}, 1190 \mathrm{~m} ; 1{ }^{ð}$, W de Puente Ixtla, 20-VII-2006, V. H. 
T., A. M. C.; 1 ठ઼, Cocoyotla, 7-X-2000, H. B., E. B.; 1 ð, Alpoyeca, 6-IX-1980, E. B.; 1 ठૈ, Amacuzac, 19-I-1990, H. B., E. B.; 1 ㅇ, Villa Ayala, 17-X-1971, H. B.; 1 , $3 \mathrm{Km}$ al SE de Cd. Ayala, 4-V-1991, H. B., E. B.; 1 ㅇ, Km 49 Cuernavaca-Taxco, 19X-1988, H. B., E. B., J. B. S.; 2 ð, Oaxtepec, 21-I-1979, H. B.; 2 ð, Xochicalco, 25II-1977, E. B.; 1 ㅇ, Tetela del Volcán, 30-VI-1982, A. I. NAYARIT: 3 ㅇ, 3 ㅇ, Río Santiago, Las Adjuntas II, 13-17-IX-1991, R. B., E. B.; 4 ㅇ, 3 ㅇ, Río las Adjuntas II, 26-XI-1991, R. B., E. B.; 4 ㅇ, 3 ㅇ, Río Santiago, Las Adjuntas, 11-13-IX-1991, R. B., E. B.; 3 o, 1 , Río Santiago, Colorado de la Mora, Arroyo San Pablito, 30-XI1991, R. B, E. B.; 2 đ̃, Río Santiago, Colorado de la Mora, Arroyo San Pablito, 28XI-1991, R. B, E. B.; 1 ð, Río Santiago Colorado de la Mora, 16-II-1992, R. B., E. B.; 9 đ, Río Santiago, Paso de Bueyes, 29-XI-1989, A. C., 220m; 1 đૈ, Río Huaynamota, Los Sabinos, 3-13-IX-1991, R. B., E. B.; 1 ð, Río Huaynamota, Los Sabinos, 27-XI-1991, R. B., E. B.; 1 §, 1 ㅇ, Unión Ríos Santiago y Huaynamota, Las Adjuntas II, 27-28-XI-1991, R. B., E. B.; 1 , Rincón de Guayabitos, 19-VIII-1981, R. Z.; 1 ㅇ, Rincón de Guayabitos, 18-VIII-1981, R. Z.; 2 §, Rincón de Guayabitos,

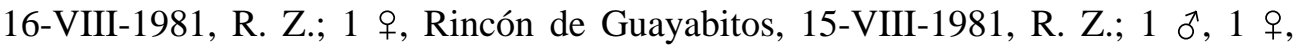
Rincón de Guayabitos, 17-VIII-1981, R. Z.; 2 ð ,4 ㅇ, Jesús María, 22-X-1989, A. C.; 1 đ̊, 5 o , Jesús María, 21-X-1989, A. C.; 3 ð 5 q, Jesús María, 18-X-1989, A. C.; 1

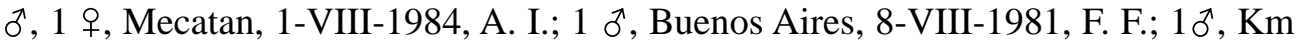
15 carretera Mecatan-Sta. Cruz, 30-VII-1984, E. B.; 1 ㅊ, 1 우, P. H. Aguamilpa, San Rafael, Arroyo la Virgen, 11-XI-1991, R. B., E. B.; 1 ð, Sierra de San Juan Km 14 carretera Alcora, 17-X-1989, A. C.; 2 우, 5 ð, 20 Km NO Acaponeta, 8-VII-1982, H. B.; 1 ㅇ, Tepic, Otate, 31-VII-1975, H. A.; 1 ㅇ, San Blas, 8-VIII-1979, E. M.; 1 ơ, 2 ㅇ, San Blas, Km 4 carretera 15, 29-VII-1984, M. G.; 1 ð̋, San Francisco, 17-X-1989, A. C. NUEVO LEON: 1 ठ , Monterrey, Arroyo de la Silla, 20-XI-1969, E. A.; 1 ð, 1 + , Linares, 17-XI-1977, E. M., E. B.; 2 ð, Cola de Caballo, 22-XI-1985, F. A., L. C. OAXACA: primer estadio 18 ninfas, segundo estadio 16 ninfas, tercer estadio 14 ninfas, cuarto estadio 1 ninfa, quinto estadio 6 ninfas, 0 , 우, Chazumba, Km $16 \mathrm{SE}$ de Chazumba, 4-V-2005, E. E., R. G, L. C.; 1 오, Teotitlan del Valle, 17-X-1978, H. B.; 1 오, Huajuapan de León, 17-IX-1980, H. B.; 1 우, Huajuapan de León, 18-IX1980, H. B.; 1 ð, Huajuapan de León, 26-X-1978, H. B.; 1 ð, 1 ㅇ, Km 31 carretera Huajuapan, Pinotepa Nacional, 29-I-1993, E. B., C. M.; 1 đ, Km 15 Izúcar de

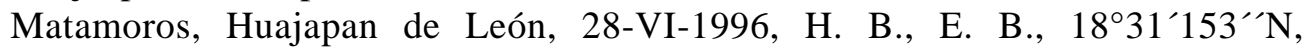
98 '25’01“'O, 4100m; 2 ð’, 1 ㅇ, Km 10 El Camarón-San Carlos, Yautepec, 31-III2000, E. B.; 2 ð, 3 ㅇ, Km 4 al E de San Carlos Yautepec, 15-VII-2000, A. I., C. M., E. B., 800m; 2 ð, Km 15 El Camarón-San Carlos Yautepec, 15-VII-2000, C. M., E. B., A. I.; 1 q, $4 \mathrm{Km}$ al E de San Carlos Yautepec, 13-VII-2000, C. M., E. B.; 1 ㅇ, 3 Km al N del Camarón, 30-VII-1996, H. B., E. B.; 1 ㅇ, 5 Km SE El Camarón, 1-VII-

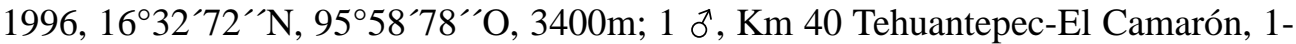
VII-1996, H. B., E. B., $16^{\circ} 24^{\prime} 62^{\prime \prime} \mathrm{N}, 95^{\circ} 37^{\prime} 72^{\prime \prime} \mathrm{O}, 1100 \mathrm{~m} ; 2{ }^{\star}$, Km 58 
Tuxtepec-Oaxaca, 28-V-1987, E. B.; 1 ㅇ, Km 16 Jalapa de Díaz Tuxtepec, 18-III1989, L. C.; 1 ㅇ, Km 5 Oaxaca-Guelatao, 29-VI-1996, H. B., E. B.; 2 ð, Km 6 Oaxaca-Guelatao, 29-VI-1996, E. B., $17^{\circ} 06^{\prime} 37^{\prime \prime} \mathrm{N}, 96^{\circ} 35^{\prime} 56^{\prime \prime} \mathrm{O} ; 1$ ㅇ, Km 40 Oaxaca-Guelatao, 19-IX-1989, E. B.; 1 ð, Monte Alban, 16-VI-1984, R. M.; 1 우, Monte Alban, 29-V-1987, F. A; 1 ㅇ, Puerto Ángel, La Galera, 17-VI-1982, L. T.; 1 , Km 127 Oaxaca-Puerto Ángel, 30-V-1987, F. A; $2{ }^{\nwarrow}, 2$ ㅇ, La Galera, 16-VI-1982, E. B., L. T.; 1 đ , Km 42 Puerto Ángel, 29-V-1985, E. M.; 2 ․, Teotitlán del Valle, 19X-1980, H. B.; 1 ð, 5 q, Teotitlán, Río Xiquilla, 5-XI-1988, E. B., L. C., R. B.; 5 ðૈ, 1 우, Km 50 Puerto Escondido-Oaxaca, 11-VI-2001, E. Barba, R. M.; 1 ð, 3 오, Km 8 Puerto Escondido-Oaxaca, 10-VI-2004, E. B., R. M.; 2 ㅇ, Km 11 Puerto

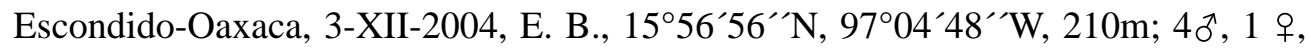

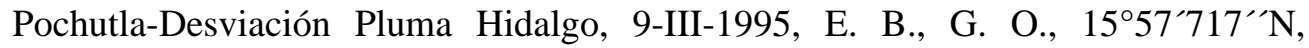

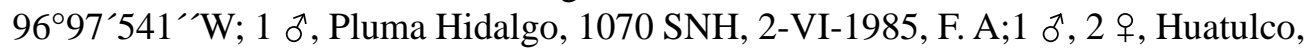

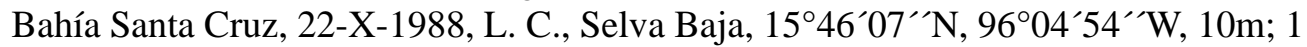
, Huatulco, Tangolunda, 30-VI-2009, H. B., W. S.; 1 đo, Tehuantepec, 22-XII-1980, H. B.; 2 ð, 3 ㅇ, San Marcos, Arteaga, 29-I-1993, E. B., C. M.; 1 ð, 2 ㅇ, Portillo del Rayo, 1-VI-1985, E. B., 1300m; 4 §ึ, 6 오, Dominguillo, 20-VIII-1998, A. R., $17^{\circ} 38^{\prime} 907^{\prime \prime} \mathrm{N}, 96^{\circ} 54^{\prime} 703^{\prime \prime} \mathrm{W}, 760 \mathrm{~m}$; 1 우, San Juan de Lues, 14-VI-1984, M. G.; 1 ð’, Huajolotitlan, 15-VII-1992, E. B., C. M.; $1 \delta^{\lambda}, \mathrm{Km} 2$ San Juan Guichicori Zacatal,

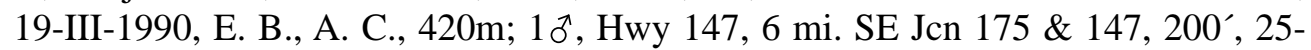
VIII-1982, C. W. O’Brien., L. O’Brien, G. W.; 1 ð’, Pluta de Oro, 31-IX-1990, E. B., A. C.; 1 ㅇ, Km 120 Sayula-La Ventosa, 19-I-1983, M. V.; $1 \delta^{\widehat{ }}$, Municipio Valle Nacional, San Mateo Yetla, 13-X-1990, E. B., E. R., A. C.; 1 으, Oaxaca, 15-VII2004, W. S.; 2 đ, 1 ․ Km 7 al W de Díaz Ordaz, 29-VI-1996, E. B., H. B., $17^{\circ} 01^{\prime} 49^{\prime \prime} \mathrm{N}, 96^{\circ} 28^{\prime} 20^{\prime \prime} \mathrm{O}$; 5 웅, Chila, 31-V-1987, L. C., E. B., F. A; 1 ð’, Camelia Roja, 2-V-1975, J. B., colecta nocturna; 1 ð, Macuilxochilt, 10-V-1985, E. B.; 1 ð, Km 15 El Carmen-San Carlos, 15-VII-2000, E. B., C. M., A. I., 800m; 1 q, Km 20

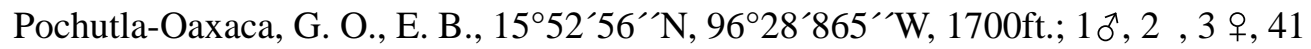
Km Puerto Escondido-Sola de la Vega, 4-VII-1990, E. B., A. C.; 1 ðૈ, Putla de Guerrero, 3-IX-1990, E. B., A. C.; 1 ㅇ, Santa María Zacatepec, 15-VII-2004, L. C., A. D., C. M., S. G., $16^{\circ} 42^{\prime} 25^{\prime \prime} \mathrm{N}, 97^{\circ} 59^{\prime} 776^{\prime \prime} \mathrm{W}, 315 \mathrm{~m}$, en Ficus insípida; 1 ㅇ, Santo Domingo Zanatepec, 23 mi E Zanatepec, 03-VI-1974, W. O`Brien, L. O’Brien, G. B. M., (IEXA); 1 \&, Oaxaca-Pinotepa Nacional Río de Arena, 14-VII-2004, L. C., A. D., C. M., S. G., $16^{\circ} 20^{\prime} 051^{\prime \prime} \mathrm{N}, 98^{\circ} 00^{\prime} 551^{\prime \prime W}$; 1 옹 San José Pacífico, cabañas "Puesta de Sol”, 8-VI-2004, E. M.; 1 ð, San Gabriel Mixtepec, Las Acacias, A. D., A. C.,

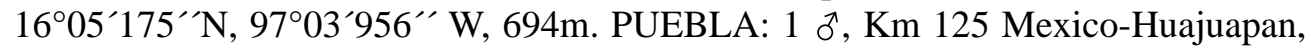
$2 \mathrm{Km}$ al P del Rijo, 26-III-1994, E. B., G. O.; 8 §, 9 ㅇ, Km 125 Mexico-Huajuapan, $2 \mathrm{Km}$ al O del Rijo, 26-I-1994, E. B., G. O.; 1 ðె, 1 ㅇ, Km 94 Cuautla-Huajuapan,

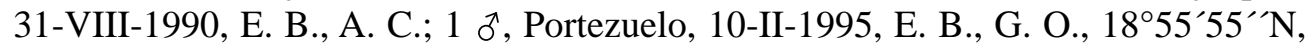
98²0`40”W, 6100 ft.; 1 ㅇ, Teyuca, 28-X-1994, C. M., H. B.; 1 ㅇ, Xochimilco, 12- 
II-1995, E. B., C. M.; 1 ㅇ, 1 ठ, Puebla-Atlixco, La Soledad, 21-III-1994, G. O., E. B.; 2 万, 1 옹, Puebla-Atlixco, Puente los Molinos, 21-III-1994, G. O., E. B.; 1 ㅇ, La Trinidad, Atlixco-Izúcar de Matamoros, 21-III-1994, G. O., E. B.; 2 ㅇ, Km 20 Puebla-Atlixco, 10-III-1994, E. B., C. M., 1990m; 1 q, 6 Km S de Coactepec, Km 42 Atlixco-Izúcar de Matamoros, 27-I-1995, G. O., E. B.; 1 \&, Tetela de Matamoros, Atlixco-Izúcar de Matamoros, 27-I-1995, E. B, G. O.; 7 đ઼, 3 ㅇ, $2 \mathrm{Km}$ al S de la

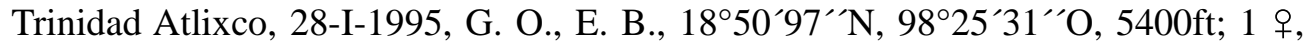
Atlixco, 11-IX-2009, H. B., E. B.; 7 §, 3 ㅇ, Atlixco, La Trinidad, 23-IV-1994, H. B.,

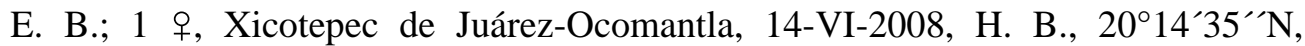
9753`36”W, 735m; 1 우, Km 10 Xicotepec-Patla, 2-VII-1994, G. O., E. B.; 1ð, Km 1 Tecamachalco-Tehuacán, 12-VI-1992, H. B., E. B.; 1 đo Tehuacán (pollos), 12-VI1992, E. B., H. B.; 1 ㅇ, Tehuacán Teotitlán del Camino, 22-VII-1992, E. B., C. M.; †, Tehuacán, San Francisco de la Cañada, 13-III-1992, H. B, E. B.; 1ð, 2 ㅇ, La Trinidad, 9-III-1994, C. M., E. B., 1680m; 1 क, La Trinidad, 28-X-1994, H. B., C. M;

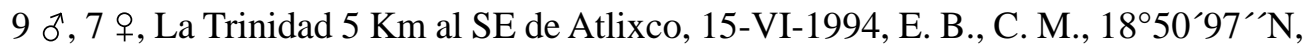
98 25’34”W; 1 오, Patla, 6-VIII-1995, H. B.; 1 đ, Patla, 13-VII-1975, colecta nocturna, H. B.; 2 ઠ, 4 +, Km 5 Pahuatlan, La Cruz, 5-VIII-1995, H. B.; 1 ๙, Atlimeya (cercano a Atlixco), 12-IX-2009, H. B., E. B.; 5 o, 8 ㅇ, Chiautla, Km 21 Chiautla-Chila de la Sal, 15-X-2006, L. C., C. M., $18^{\circ} 12^{\prime} 23^{\prime \prime} \mathrm{N}, 98^{\circ} 32^{\prime} 22^{\prime} \mathrm{W}$,

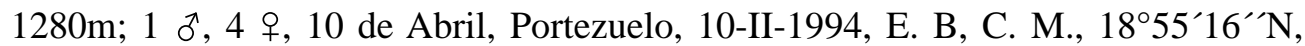

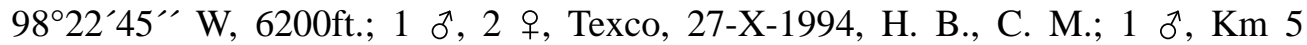
desviación Sta. Ana, 11-III-1994, E. B., C. M.; 1 \&, Paijatlan Lecho del Río, 10-VII1994, E. B., C. M., 700m; 1 , Coxcatlan, 17-I-1992, H. B, E. B., C. M., G. O; 1 ठ, Axusco, Río Calsos, 4-XI-1988, E. B., R. B., L. C.; 1 ㅇ, Cuetzalan, 2-VIII-1986, H. B.; 2 đ, 6 o, Telpatlán, 3-XI-1988, R. B., E. B., L. C.; 3 ㅇ, Km 9 camino a Santa Ana, 11-III-1994, E. B., C. M.; 1 ð, 1 क, Tilapa, 18-X-1978, H. B.; 2 ð, 1 , 8 Km N de Acatlán, 11-VII-1999, H. B., E. B., 1290m; 1 ðત, Km 25 Los Ángeles Tetela-

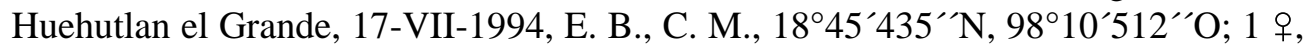
Puerto El Gato, 11-VII-1999, H. B., E. B., 1150m; 1 ð, 5 Km SW Petlalcingo, 11VII-1999, E. B., H. B.; 1 ठ઼, San Esteban Necoxcalco, 22-VII-1992, E. B., C. M.; 1

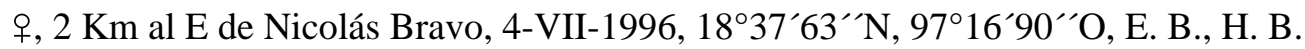
QUERETARO: 3 ð, 2 ㅇ, Km 5 Tilaco, Agua Zarca, 21-VII-1995, E. B., C. M., $21^{\circ} 10^{\prime} 886^{\prime \prime} \mathrm{N}, 99^{\circ} 13^{\prime} 343^{\prime} \mathrm{W}, 1070 \mathrm{~m}$; 1 ð。, Km 8 Agua Zarca-La Neblina, 28-V1998, E. B., $21^{\circ} 25^{\prime} 214^{\prime \prime} \mathrm{N}, 9^{\circ} 05^{\prime} 296^{\prime \prime} \mathrm{O}, 1090 \mathrm{~m}$; $1{ }^{\star}$, Puente de Dios, Laguna de Tepisquintla, Municipio de Jalpan, E. B., H. B., 21 $11^{\prime} 271^{\prime \prime} \mathrm{N}, 99^{\circ} 30^{\prime} 695^{\prime \prime} \mathrm{W}$, 1040m; 1 ๙, 2 우, Ciudad de Querétaro, 8-15-IV-2009, K. B.; 1 으, Ciudad de Querétaro, 5-16-I-2009, K. B.; 1 ð, Ciudad de Querétaro, 29-XI-2008, H. B.; 1 ठ, La Venta-Lira, 12-IX-2007, H. B., E. B.; 3 ð, 3 ․, Chuveje, 21-VI-1998, L. C., G. O.; 1 ㅇ, 2 Km N Chuveje, 18-VIII-1998, H. B., E. B.; 1 ð, 1 ㅇ, Km 8 Neblinas-Agua Zarca, 23-VI-1998, L. C., G. O.; 1 +, Km 12 Pinal-Misión de Bucareli, 27-IV-1998, 


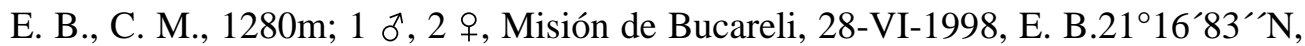
99 36'46”O, 800m. QUINTANA ROO: 1 우 , Carrillo Puerto, 28-31-VII-1964, J. C. P., D. Pallister. SAN LUIS POTOSI: 1 ð, Cd. Valles, 20-V-1978, G. O.; 2 ð, 2 ㅇ, Río Micos, 13-IV-1980, H. B.; 6 ð, 7 ㅇ, Tamazunchale, 21-V,-1975, colecta nocturna, H. B.; 4 ð̃, 3 o, Tierra Quemada Km 143 Querétaro-San Luis Potosí, 21-VIII-2006, H. B., E. B., $21^{\circ} 40^{\prime} 04^{\prime \prime} \mathrm{N}, 100^{\circ} 41^{\prime} 49^{\prime \prime} \mathrm{W}, 1800 \mathrm{~m}$; 1 ㅇ, $12 \mathrm{Km}$ E de Cerritos, 3-4-VIII2006, H. B., E. B., $22^{\circ} 29^{\prime} 48^{\prime} \mathrm{N}, 100^{\circ} 25^{\prime} 05^{\prime} \mathrm{W}, 1644 \mathrm{~m} ; 1$ ð’, Km San Luis PotosíRio Verde, Puente El Morenito, 24-VIII-2006, H. B., E. B., $22^{\circ} 05^{\prime} 49^{\prime \prime} \mathrm{N}$, $100^{\circ} 45^{\prime} 33^{\prime \prime} \mathrm{W}, 1973 \mathrm{~m}$; 2 ㅇ, Tamazunchale, 22-V-1975, H. B., colecta nocturna; 2 ð , 4 mi NE Xitla, V-26-1974, O’Brien, G. B. M. SINALOA: 1 ð̊, Concordia, Km 235 Durango- Mazatlán, L. C., D. B. $23^{\circ} 24^{\prime} 51^{\prime \prime} \mathrm{N}, 105^{\circ} 52^{\prime} 01^{\prime \prime} \mathrm{W}, 1196 \mathrm{~m}$; 1 우, Panititlan, 21-X-1982, M. G.; 1 ㅇ, El Pinito, 8-VIII-1997, 500m, 1 ㅇ, 10.4 mi S Elota, Rt. 15, 23-VIII-1964, P. Rauch, (UCRC); SONORA: 1 ð, Guasaremos, Río Mayo, 2-VII-1935, L. G. G. TABASCO: 1 ð, 3 ㅇ, Villahermosa, Agua Blanca, 17VI-1989, A. C., L. C. TAMAULIPAS: 1 ð, Gómez Farías camino a Ejido Azteca, 15IV-2003, L. C., I. P., en Ficus cotinifolia; 2 ð, 1 ㅇ, Gómez Farías, Ejido Azteca,

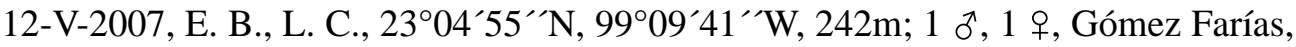

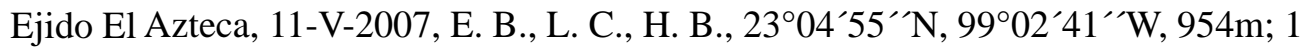
ㅇ, Gómez Farías Km 5 a Alta Cima, 12-V-2007, H. B., E. B., L. C., 230244”N,

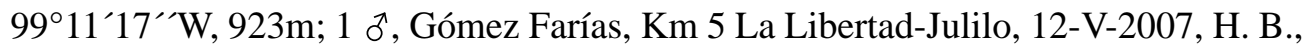

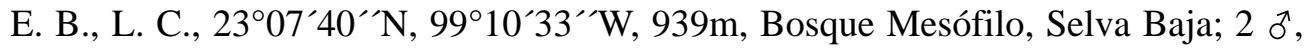
Gómez Farías, Rancho El Cielo, 29-IX-1983, M. G., A. I.; 1 đૈ, Gómez Farías, Camino a Vicente Guerrero, 27-III-2001, L. C., N. Peñaloza, (IEXA); 8 §, 6 ㅇ, Altamira Km 49 Tampico-Ciudad Victoria, 9-V-2007, H. B., E. B., L. C., $22^{\circ} 31^{\prime} 49^{\prime \prime} \mathrm{N}, 98^{\circ} 07^{\prime} 49^{\prime \prime} \mathrm{W}, 138 \mathrm{~m}$, encinar; 1 o $^{`}, 3$ ㅇ, Llera de Canales, Km 11 Llera-

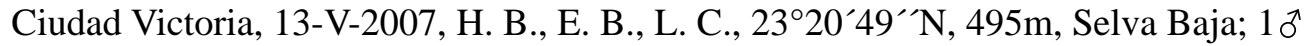

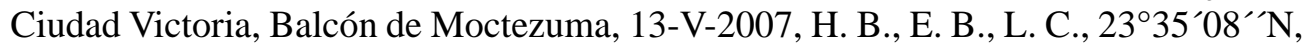

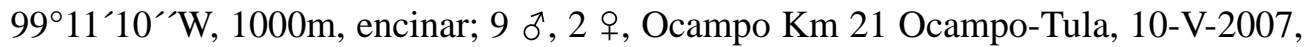
H. B., E. B., L. C., $22^{\circ} 54^{\prime} 28^{\prime \prime} \mathrm{N}, 99^{\circ} 30^{\prime} 21^{\prime \prime} \mathrm{W}, 648 \mathrm{~m}$, encinar; 3 ð。, Tula Km 20 Tula-Ocampo, Colonia Guadalupe, 12-V-2007, H. B., E. B., L. C., 22 ${ }^{\circ} 53^{\prime} 13^{\prime}{ }^{\prime} \mathrm{N}$, 99²8`34”W, 446m, Selva Baja; 2 ð, Ocampo, Aniceto Medrano, 10-V-2007, H. B., E. B., L. C., $22^{\circ} 55^{\prime} 33^{\prime \prime} \mathrm{N}, 99^{\circ} 31^{\prime} 05^{\prime \prime} \mathrm{W}, 899 \mathrm{~m}$. TLAXCALA: $10^{\star}, 1$ ㅇ, Amaxac de Guerrero, Atlihuetzian, 27-VIII-1992, H. B., E. B. VERACRUZ: 2 ㅇ, Fortín de las Flores, Barranca de Metlac, 31-I-1976, H. B.; 1 ð, 1 \%, Metlac, 10-VII-1975, colecta nocturna, H. B.; 1 ㅇ, Metlac, 4-II-1978, E. M.; 1 ð, Barranca de Metlac, 28-VII2000, E. B., C. M.; 1 ð, Barranca de Metlac, 12-VII-2000, E. B., C. M.; 1 ð, 1 ㅇ, Barranca de Metlac, 27-VII-2000, E. B., A. I.; 2 ㅇ, Barranca de Metlac, 31-I-1976, H. B.; 1 올 Estación Biológica Los Tuxtlas, 27-IV-1991, G. O., C. M.; 1 ㅇ, Estación

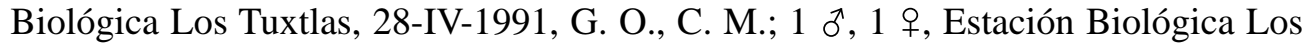
Tuxtlas, 20-IX-1991, R.; 2 ㅇ, Los Tuxtlas, 1-3-IV-1993, H. B.; 1 ð, 2 ㅇ, Estación 
Biológica Los Tuxtlas, 26-IV-1991, G. O., C. M.; 1 ㅇ, Estación Biológica Los Tuxtlas, 17-IX-1987, F. A.; 1 , Los Tuxtlas, 18-V-1983, G. B. M.; 1 ㅇ, Los Tuxtlas, 30-VII-1989, S. Z.; 1 ㅇ, Zongolica, 30-VII-1967, L. R.; 2 ㅇ, Km 7 OrizabaZongolica, 26-VII-2000, E. B., A. I., 1180m; 1 우, Zongolica, 26-V-1967, L. R.; 1 ठ , Xalapa, 6-VI-1984, J. P.; 1 ð, Xalapa, 18-VI-1984, J. P.; 1 q, Xalapa, VII-1985, J. P.; 1 오 Ixtaczoquitlán "Los Sifones", 26-VII-2000, A. I., E. B., 1000m; 1 đ, 1 ㅇ, Orizaba Ixtaczoquitlán, 4-V-2000, E. B., A. I., 90m; 1 ㅇ, Buena Vista Municipio Ixtaczoquitlán, 28-VII-2000, E. B.A. I., 1000m; 1 ㅇ, Ixtaczoquitlán, 13-IV-2000, H. B., E. B.; 1 ð, Orizaba, Jalapilla, 2-V-2000, E. B.A. I.; 1 ð, Orizaba, Jalapilla, 5-I2000, H. B., E. B., 950m; 1 ð, Dos Caminos, 16-VIII-1975, L. R.; 1 ð, Dos

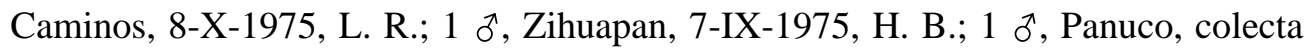
nocturna, 28-VIII-1975, H. B.; 2 \%, Tolome, 7-XI-1974, trampa de luz, 20 m, H. B.; 3 ð઼, 3 q, Puente Nacional, 9-XI-1974, colecta nocturna, H. B., 140 m; 1 ㅇ, Tecolapa, 9-IX-1977, E. B.; 1 \&, Huatusco, colecta nocturna, 20-III-1975, M. A. M.; 1 \&, San Andrés, Tuxtla, 1-X-1970, E. B.; 1 đ̃, Plan del Río, 1-II-1978, H. B.; 1 q, 4 Km al Norte de Cardel, 9-III-1989, H. B.; 1 ð 1 우, Km 28 Federal Puebla-Orizaba, 14-II1984, M. G., V. M.; 1 ð, Tempoal, colecta nocturna, 29-VIII-1975, H. B.; 1 ð, Atoyac, 28-VII-2000, E. B., A. I., 350m; 1 क, Atoyac, 28-VII-2000, E. B., A. I., 550m; 1 ․ Actopan, La Mancha, 14-VII-2000, L. C., colecta nocturna; 1 ㅇ, La Mancha, 30-I-1982, L. T.; 1 ㅇ, Casitas, 22-XII-1998, K. B., H. B.; 1 오, Estación Microondas, San Juan Fortín de las Flores, 3-V-2000, E. B., A. I., 1150m; 1 +, Km 4 Atzalan-Martínez de la Torre, 25-V-2000, E. B., H. B., 1450m; 1 을 Orizaba, Buena Vista, 3-V-2000, E. B., A. I., 1200m; 1 ð 1 우, Cerro Aparicio 27 Km Sur de Casitas, 26-V-2000, E. B., H. B., 100m; 2 ㅇ, Nanchital, 5-X-1976, H. B., colecta nocturna; 1 §, Coatepec, 11-VII-1975, H. B., Nocturna; 1 , Nautla, 13-VII-1975, H. B.; 1 ㅇ, Huatusco, 21-III-1975, E. B.; 1 ð, Plan del Río, 1-II-1978, E. B.; 1 ð, Playa Norte, 23-I-1957, H. B.; 1 q, Veracruz, 20-V-1962, H. B. YUCATAN: 1 q, Dzibilchaltun, 26-VIII-1964, J. C. P., D. Pallister; 2 우, 1오 $23 \mathrm{Km}$ al N de Valladolid en Acromia sp., V-1994, E. B.; 1 ㅇ, Chichenitza, 13-IX-1994, E. B., 20³8 $004^{\prime \prime} \mathrm{N}$, 88²7’082”W, 700ft.; 1 ㅇ, Km 30 Muna-F. Carrillo Puerto, 21-VI-1989, A. C., L. C.;

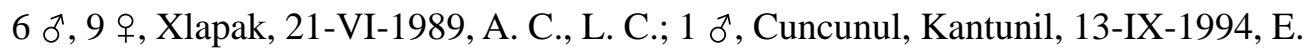

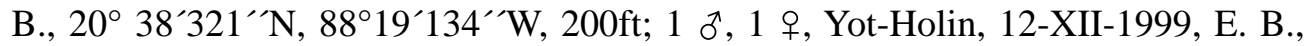

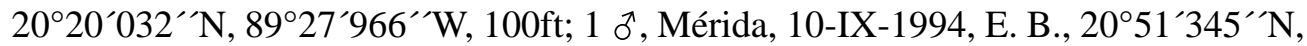
$89^{\circ} 44^{\prime} 580^{\prime \prime} \mathrm{O}, 100 \mathrm{ft}$.

ZACATECAS: 2 ㅇ, Felipe Pescador, Cañitas, 23-XI-1995, E. B., H. B.; 1 đ`, Zacatecas, 15-X-1982, A. I.

\section{DISCUSIÓN}

De las tres especies reportadas para México, F. multispinus, es relativamente delgada, lóbulo anterior pronotal, escutelo y vientre, café oscuro a café rojizo, fémur anterior 
presentando manchas cafés. F. piligera, es la más robusta, cabeza, lóbulo pronotal escutelo y superficies ventrales negro mate, hemiélitro de café oscuro a negruzco, con los márgenes laterales pálidos y usualmente puntos amarillos pálidos cerca del área apical interior del corium, hilera doble de espinas en ambas especies bien definidas, F.vicinalis, es más pequeña que $F$. piligera, cabeza, lóbulo anterior del pronoto, escutelo, membrana hemelitral, negros; ápice del escutelo, corium, y patas en general ocre, lóbulo posterior del pronoto pardo oscuro, con márgenes laterales rojizos, hilera doble de espinas no tan bien definidas como en las especies anteriores. En general la especie más abundante y de más amplia distribución es $F$. piligera.

AGRADECIMIENTOS. Agradecemos a la M. C. Cristina Mayorga del Instituto de Biología (UNAM), por todas las facilidades al momento de revisar el material depositado en la Colección de Insectos, así mismo agradecemos a C. H. Ramos, del Instituto de Biología (UNAM) por la identificación de algunas plantas hospederas. Parte del material fue colectado con financiamiento de los siguientes proyectos: CONACYT (34238-V) "Lygaeidae (Hemiptera-Heteroptera) asociados a Ficus spp. (Moraceae) y su efecto en la depredación de semillas en la región del Golfo de México"; y DGAPA (IN223503-3) "Análisis comparativo de la coreidofauna en tres estados de la Republica Mexicana: Guerrero, Oaxaca y Chiapas (Hemiptera-Heteroptera)".

\section{LITERATURA CITADA}

Alayo, P. D. 1973. Los Hemipteros de Cuba. Parte XI. Familia Lygaeidae. Torreia (N.S), 25: 1-79

Baranowski R. M. \& J. A. Slater. 2005. The Lygaeidae of the West Indies. University of Florida. Florida Agricultural Experiment Station.Florida. 266p.

Cervantes, L. 2005. Lygaeidae (Hemiptera-Heteroptera) asociados con Ficus spp. (Moraceae) en el área de la Reserva de la Biosfera El Cielo, Tamaulipas. Pp. 367-388. In: G. Sanchéz-Ramos, P. Reyes-Castillo y R. Dirzo (eds.). Historia Natural de la Reserva de la Biosfera El Cielo, Tamaulipas, México. Universidad Autónoma de Tamaulipas. México.

Harrington, B. J. 1980. A generic level revision and cladistic analysis of the Myodochini of the world (Hemiptera, Lygaeidae, Rhyparocrhominae). Bulletin of the American Museum of Natural History, 167(2): 45-116.

Henry, T. J. \& R. C. Froeschner. 1988. Catalog of the Heteroptera, or True Bugs, of Canada and the Continental United States. E. J. Brill, United States of America, New York. 958p.

Slater, J. A. 1964. A catalogue of the Lygaeidae of the World. University of Conneticut Storrs, Conneticut. $211 \mathrm{p}$.

Slater, J. A. \& R. M. Baranowski. 1990. Lygaeidae of Florida (Hemiptera: Heteroptera). Florida Department of Agriculture and Consumer Services, Doyle Conner, Comissioner. Florida. 14: 211 p.

Slater, J.A. \& H. Brailovsky. 2000. Lygaeidae (Hemiptera) In: Biodiversidad, Taxonomía y Biogeografía de Artrópodos de México: Hacia una Síntesis de su Conocimiento. Llorente, B.J.E., González, E.S. y Papavero, N. (eds.). Universidad Nacional Autónoma de México, Facultad de Ciencias: $319-333$. 